\title{
Exploring the Climatic Response to Wide Variations in Ocean Heat Transport on an Aquaplanet
}

\author{
M. CAMERon Rencurrel AND Brian E. J. Rose \\ Department of Atmospheric and Environmental Sciences, University at Albany, State University of New York, Albany, \\ New York
}

(Manuscript received 14 December 2017, in final form 30 April 2018)

\begin{abstract}
The climatic impact of ocean heat transport (OHT) is studied in a series of idealized aquaplanet climate model experiments. OHT is prescribed through a simple geometrical formula spanning a wide variety of amplitudes and meridional extents. Calculations with a comprehensive GCM are compared against a simple diffusive energy balance model (EBM). The GCM response differs from the EBM in several important ways that illustrate linkages between atmospheric dynamics and radiative processes. Increased OHT produces global mean warming at a rate of $2 \mathrm{~K} \mathrm{PW}^{-1} \mathrm{OHT}$ across $30^{\circ}$ and a strong reduction in meridional temperature gradient. The tropics remain nearly isothermal despite locally large imposed ocean heat uptake. The warmer climate features reduced equatorial convection, moister subtropics, reduced cloudiness, and partial but incomplete compensation in atmospheric heat transport. Many of these effects are linked to a weakened Hadley circulation. Both the warming pattern and hydrological changes differ strongly from those driven by $\mathrm{CO}_{2}$. Similar results are found at $0^{\circ}$ and $23.45^{\circ}$ obliquity. It is argued that clouds, rather than clear-sky radiative processes, are principally responsible for the global warming and tropical thermostat effects. Cloud changes produce warming in all cases, but the degree of warming depends strongly on the meridional extent of OHT. The strongest warming occurs in response to mid- to high-latitude OHT convergence, which produces widespread loss of boundary layer clouds. Temperature responses to increased OHT are quantitatively reproduced in the EBM by imposing GCM-derived cloud radiative effects as additional forcing.
\end{abstract}

\section{Introduction}

A fundamental feature of the climate system is the poleward transport of energy to counteract the differential heating of Earth's surface by the sun. This total heat transport (THT) is composed of atmospheric and oceanic components. In the atmosphere, transient eddies, stationary eddies, and the mean meridional circulation (MMC) all work to transport moist static energy (MSE) poleward, whereas in the ocean, sensible heat is transported out of the tropics via surface and deep ocean currents. Observationally based estimates show the THT to be remarkably symmetric about the equator, with maxima of roughly $5.5 \mathrm{PW}$ near $35^{\circ}$ in both hemispheres. The atmospheric heat transport (AHT) component dominates poleward of $30^{\circ} \mathrm{N}$ and $30^{\circ} \mathrm{S}$, reaching a peak of $5 \mathrm{PW}$ near $43^{\circ}$ while the oceanic heat transport (OHT) is strongest in the deep tropics, with

Corresponding author: M. Cameron Rencurrel, crencurrel@ albany.edu peaks near 2.1 and $1.6 \mathrm{PW}$ at $18^{\circ} \mathrm{N}$ and $18^{\circ} \mathrm{S}$ (Trenberth and Caron 2001; Wunsch 2005; Czaja and Marshall 2006). Unlike the THT, AHT and OHT are not symmetric about the equator, with a slight northward and southward cross-equatorial transport of the oceanic and atmospheric components respectively.

While Held (2001) argued that variations in AHT and OHT are coupled through their Ekman transports, modeling studies have shown that tectonic movement of tropical landmasses can give rise to significant changes in OHT, partly through modulations of the tropical oceanic static stability (i.e., depth of the tropical thermocline) (Bice et al. 2000; Enderton and Marshall 2009; Ferreira et al. 2011). Stone (1978) showed that THT is set by astronomical parameters and albedo and is largely insensitive to variations in AHT and OHT. This implies that any increase in the OHT must be accompanied by a compensating decrease in the AHT to maintain equilibrium. This compensation has been a constant presence in the modeling community, in both past simplified models (Bjerknes 1964; Manabe 1969) and recent, more 
advanced coupled general circulation models (GCMs; e.g., Yang et al. 2015).

OHT is driven by both tropical shallow wind-driven gyres and overturning cells as well as cross-equatorial deep circulations. These processes vary on numerous different time and spatial scales (Ferrari and Ferreira 2011), ranging all the way up to the longest tectonic time scales, at which OHT is primarily determined by continental boundaries and gateways (e.g., Covey and Barron 1988; Bice et al. 2000; Enderton and Marshall 2009; Ferreira et al. 2011). Since most of these time scales are well separated from the rapid equilibration of the atmosphere, it is meaningful to study the climatic impact of OHT variations in uncoupled climate models in which OHT patterns are prescribed.

One of the outstanding problems within the climate community has been how to reconcile the geological proxy records of past warm, equable climates such as the early Eocene (56-48 Ma), characterized by both an increase in the global-mean surface temperature and a decrease in the equator-to-pole temperature gradient (Greenwood and Wing 1995; Crowley and Zachos 2000), with the numerical simulations of the same period (Barron 1987; Huber and Sloan 2001; Caballero and Langen 2005; Rose and Ferreira 2013). A key feature of these climate states is the presence of a "tropical thermostat" mechanism preventing substantial changes in equatorial sea surface temperature (SST) despite potentially large forcings (Pearson and Palmer 2000).

Previous studies of the climatic impact of OHT variations in uncoupled climate models (Winton 2003; Herweijer et al. 2005; Barreiro et al. 2011; Rose and Ferreira 2013; Koll and Abbot 2013) have consistently found that OHT warms the planet. Increased oceanic energy flux out of the deep tropics results in global mean surface warming and a reduction of the equator-to-pole temperature gradient, indicating the presence of a tropical thermostat mechanism. This warming is remarkable given that a change in OHT represents a conservative forcing that simply redistributes energy between different latitude bands. The lack of local cooling of the deep tropics is also remarkable, given that these are regions of locally enhanced ocean heat uptake. Because OHT is completely unconstrained by paleoclimate proxy data (Bice et al. 2000), it is possible that variations in OHT are a significant driver of climate change during these periods.

Global mean warming from increased OHT might be expected due to a reduction in sea ice extent and consequent decrease in planetary albedo. The sea ice margin is strongly coupled to convergence of OHT in the subpolar oceans (e.g., Bitz et al. 2005; Rose et al. 2013; Singh et al. 2017). A number of previous modeling studies have indeed found that the global climatic response to OHT variations is mediated at least in part by sea ice feedbacks (Seager et al. 2002; Winton 2003; Herweijer et al. 2005; Barreiro et al. 2011; Singh et al. 2017). This coupling is especially prominent in aquaplanet simulations with interactive sea ice compared to more realistic continental configurations (e.g., Langen and Alexeev 2004; Ferreira et al. 2011; Rose et al. 2013; Rose 2015), perhaps because of the lack of geographic muting effects of high-latitude continents (Eisenman 2010).

On the other hand, many of the above studies attributed substantial warming to radiative feedback processes unrelated to sea ice. Herweijer et al. (2005) varied the amplitude of the present-day OHT pattern in several models and attributed the largest share of the surface warming to increased clear-sky greenhouse trapping by water vapor. Rose and Ferreira (2013) took a more idealized approach, applying a wide range of magnitudes and meridional patterns of OHT to an intermediate-complexity aquaplanet model in a warm, ice-free regime. They also found warming dominated by clear-sky water vapor effects. Increased upper-tropospheric water vapor in their simulations was attributed to extratropical moist convection. Interestingly, the warming extended to the poles in all cases, insensitive to the meridional structure of the OHT. Koll and Abbot (2013) used similar idealized experiments (but more comprehensive models, though also ice-free) to study the tropical thermostat mechanisms preventing substantial changes in equatorial sea surface temperature (SST) in response to changes in OHT. They found that both decreased wind-driven evaporation and equatorial convection associated with the slowdown of the Hadley cell played a major role.

The idealized study by Rose and Ferreira (2013) was motivated by coupled simulations showing that meridional profiles of OHT are not fixed, but can vary with continental configuration and climatic state (e.g., Hotinski and Toggweiler 2003; Enderton and Marshall 2009; Ferreira et al. 2010, 2011; Rose 2015), as different modes of ocean circulation are emphasized. The purpose of this paper is to extend the work of Rose and Ferreira (2013) to a more comprehensive climate model. We will apply the same broad range of OHT patterns and magnitudes in an aquaplanet model with substantially higher vertical resolution and less idealized parameterizations of the moist physics. The aquaplanet allows us to better unravel the mechanisms driving the climate change without the complicating effects of land-sea interactions. Such models have been 

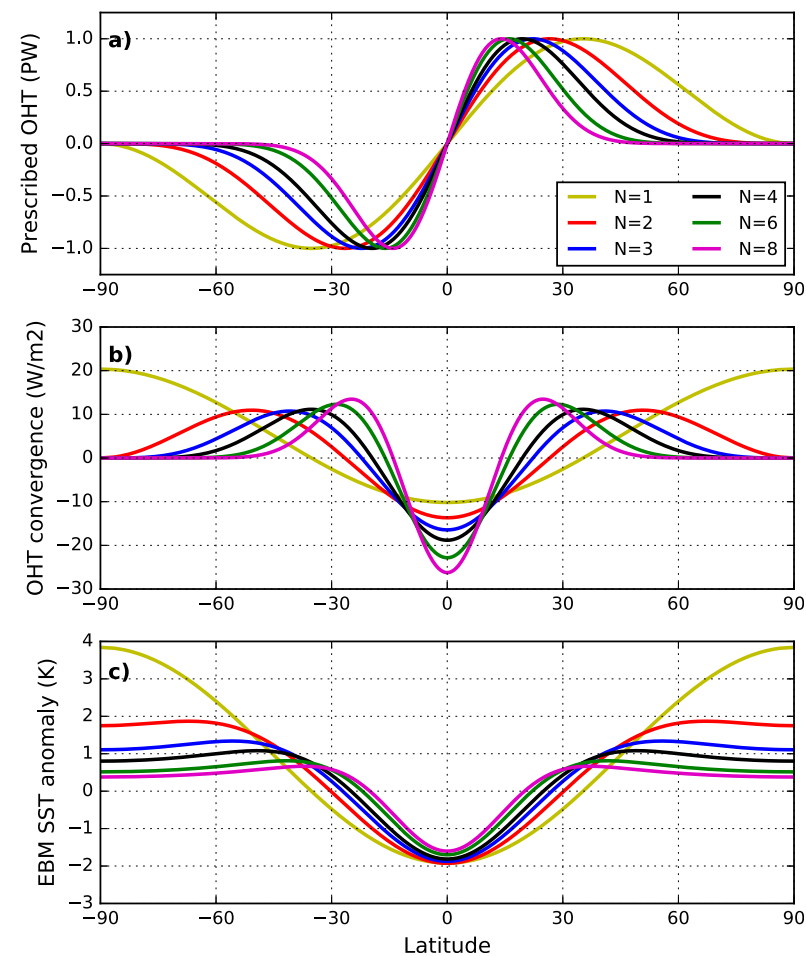

FIG. 1. (a) Idealized OHT profiles computed from (1), shown for different values of the meridional scale parameter $N$ and $1 \mathrm{PW}$ peak transport. (b) Convergence of these profiles in $\mathrm{W} \mathrm{m}^{-2}$ (the prescribed sea surface heating in our model experiments). (c) SST anomalies for a $1 \mathrm{PW}$ increase in OHT as calculated with our baseline energy balance model (2).

shown to be surprisingly Earthlike in nature (Medeiros and Stevens 2011) and a crucial step in the model hierarchy framework needed to fully understand the climate system (e.g., Held 2005; Jeevanjee et al. 2017).

To this end, we adopt the simple formula of Rose and Ferreira (2013):

$$
\mathrm{OHT}=\Psi \sin (\phi) \cos (\phi)^{2 N}=\Psi x\left(1-x^{2}\right)^{N},
$$

where $x=\sin (\phi), \varphi$ is latitude, $N$ is a positive integer that sets the meridional extent of OHT, and $\Psi$ is a constant (in units watts). Parameter $N$ ranges from 1 to 8, as shown in Fig. 1a. Parameter $\Psi$ is chosen to give maximum amplitudes ranging from 1 to $4 \mathrm{PW}$ in our simulations. This range is deliberately chosen to more than span the entire plausible range of OHT on Earth, as captured by observations (Trenberth and Caron 2001) and coupled simulations with different continental configurations and background states (Ferreira et al. 2010; Hotinski and Toggweiler 2003; Rose 2015).

Taking the convergence of (1) [see (A5) in appendix A] gives the $q$ flux prescribed in the ocean mixed layer, plotted in Fig. 1b. There is a steady surface heat sink straddling the equator, and an equal-but-opposite heat source in the subtropics (for large $N$ ) or mid- to high latitudes (for small $N$ ). The global mean forcing is $0 \mathrm{~W} \mathrm{~m}^{-2}$ in all cases. The forcing is zero at the poles in most cases. The exception is $N=1$, where maximum OHT convergence occurs at the poles. This polaramplified heating pattern will turn out to be an outlier in our set of simulation results. Such a wide range of OHT patterns has yet to be studied at this level of the climate model hierarchy.

Before introducing our GCM simulations, it is useful to define a baseline climatic response to a $1 \mathrm{PW}$ increase in OHT using a very simple energy balance model (EBM). The EBM is a one-dimensional diffusive model based on the zonal-mean perturbation energy budget:

$$
C \frac{\partial T}{\partial t}=\lambda T(\phi)+H(\phi)-\nabla \cdot F(\phi)
$$

where $T$ is a local SST anomaly, $C$ is a column heat capacity, $\lambda<0$ is a climate feedback parameter, $H(\phi)$ is the prescribed OHT convergence from (1), and $-\nabla \cdot F(\phi)$ is the AHT convergence (both in $\mathrm{W} \mathrm{m}^{-2}$ ). For our baseline model we adopt the classical dry-diffusive parameterization (e.g., North 1975a), which crucially endows the EBM with a nonlocal response to the imposed forcing. Details and parameter values are discussed in section 4 . The steady-state form of (2) forced by the convergence of (1) is analytically solvable for any $N$, as laid out in appendix A.

Figure 1c shows SST anomalies for a $1 \mathrm{PW}$ increase in OHT in the EBM. This baseline model makes several specific predictions. Global mean warming is zero as expected, given the linear nature of the EBM. The equator cools by nearly $2^{\circ}$. This cooling decreases weakly with $N$; it scales not with the local equatorial heat sink but rather its cross-tropical average (i.e., with $\mathrm{OHT}$ across $30^{\circ}$ latitude). The extratropics get warmer, with maximum warming collocated with maximum heating. Polar warming is a strongly decreasing function of $N$-it is largest for $N=1$. The tropical cooling in particular is contrary to the numerous GCM studies discussed above. Understanding why and how GCMs differ from this baseline is a crucial step toward understanding the role of OHT in the climate system.

Key questions to be addressed here are as follows. How does the GCM response to an increase in OHT differ from the baseline EBM? How robust is the warming effect of increased OHT across a broad range of specified, idealized OHT patterns, and do the mechanisms driving this warming depend sensitively on the 
spatial pattern on the OHT? What are the relative contributions of cloud versus clear-sky processes to the warming? Specifically, does the equatorial thermostat described by Koll and Abbot (2013) operate for both subtropical-scale and hemispheric-scale OHT variations? We investigate the robustness of our findings to different background climates by repeating our calculations at $0^{\circ}$ and $23.45^{\circ}$ obliquity. As we will show, the obliquity itself plays a key role in the equator-to-pole SST gradient, a result that has not been widely appreciated in the literature.

The rest of this paper is organized as follows. Section 2 describes the two configurations of our GCM. The main results are presented in section 3 , including the effects of OHT on temperatures, hydrological cycle, atmospheric circulation, and energy budgets, and a brief aside on the effects of obliquity. In section 4, we use several variants of the EBM to understand the relative importance of different warming mechanisms. Discussions and conclusions follow in section 5 .

\section{Model description}

We use an atmospheric GCM coupled to an aquaplanet mixed layer ocean with a steady, zonally symmetric prescribed heat source/sink term (the $q$ flux) representing regions of convergence and divergence of OHT, as shown in Fig. 1. The model is the Community Atmospheric Model, version 4 (CAM4), with a finite-volume dynamical core at $2.0^{\circ} \times 2.5^{\circ}$ horizontal resolution, 26 vertical levels, and a modified form of the Zhang-McFarlane deep convection scheme (Zhang and McFarlane 1995; Neale et al. 2013). The model has fully interactive clouds and water vapor and thus incorporates many of the fundamental radiative feedback processes. Reference greenhouse gases are 348 ppmv $\mathrm{CO}_{2}, 1650$ ppbv $\mathrm{CH}_{4}$, and 306 ppbv $\mathrm{N}_{2} \mathrm{O}$, with all other greenhouse gases set to zero. Ozone is prescribed as in Blackburn and Hoskins (2013). The surface albedo is fixed at 0.1. Sea ice is omitted and SST is permitted to drop below freezing. These specifications follow previous aquaplanet intercomparison protocols (Lee et al. 2008; Rose et al. 2014; Voigt et al. 2016).

We choose to exclude sea ice from these simulations because, as mentioned in the introduction, previous studies have shown that the coupling between sea ice and OHT convergence patterns is extraordinarily strong in aquaplanet simulations (e.g., Rose 2015). We wish to study the effects of OHT on atmospheric radiative and dynamical processes without the confounding effect of very large changes in sea ice extent.

We use two different control simulations, both with the $q$ flux set to zero, but with obliquity set to $0^{\circ}$ or $23.45^{\circ}$. The $0^{\circ}$ or perpetual equinox model has a $10-\mathrm{m}$ mixed layer depth and is identical to the multimodel comparison analyzed in Rose et al. (2014) and Rose and Rencurrel (2016). The $23.45^{\circ}$ obliquity model has seasonally varying insolation and a mixed layer depth of $60 \mathrm{~m}$, designed to replicate the setup used in Rose and Ferreira (2013). The $0^{\circ}$ simulations were run for 30 years, with averages computed over the last 20 . The $23.45^{\circ}$ simulations were run for 60 years to account for longer equilibration times associated with the increased mixed layer depth.

SST profiles for the two zero $q$-flux control simulations are shown as black lines in Fig. 2. Global mean temperatures are 288.57 and $291.70 \mathrm{~K}$ for $0^{\circ}$ and $23.45^{\circ}$ obliquity, respectively. The majority of the temperature difference is in the polar regions, as the $0^{\circ}$ model has zero insolation at the poles and SST is allowed to drop below freezing. We will return briefly to these obliquity-induced temperature differences below in section $3 b$.

\section{Results}

\section{a. Surface temperature}

Increasing OHT out of the tropics results in a global mean warming and a reduced equator-to-pole SST gradient in all cases. Figure 2 shows a representative subset of the results for $N=1,2$, and 6. Like previous results (Rose and Ferreira 2013; Koll and Abbot 2013; Herweijer et al. 2005), these increases are characterized by nearly invariant equatorial SSTs, extratropical SSTs that depend strongly on the pattern of OHT convergence. This is different than the EBM calculations shown in Fig. 1c, which were characterized by a tropical cooling. Figure 2 also shows the effects of doubling $\mathrm{CO}_{2}$, which warms at all latitudes in a weakly polar-amplified pattern, resulting in a global mean warming of 1.71 and $1.97 \mathrm{~K}$ for $0^{\circ}$ and $23.45^{\circ}$ obliquity, respectively.

The lack of tropical cooling in response to a locally large oceanic energy sink is remarkable. In fact, equatorial SSTs actually warm upward of $2 \mathrm{~K}$ in response to large increases in OHT. This, along with the baseline EBM results, implies that the climate system adjusts to the increased OHT by moving energy back into the tropics. Understanding what drives this "tropical thermostat" is key to understanding why OHT warms the planet. Away from the equator, maximum surface warming is usually collocated with the maximum surface heating. The warming extends to the poles only for $N=1$ (for which the poles are directly heated) and $N=2$ (peak heating near $50^{\circ}$ latitude). This differs 

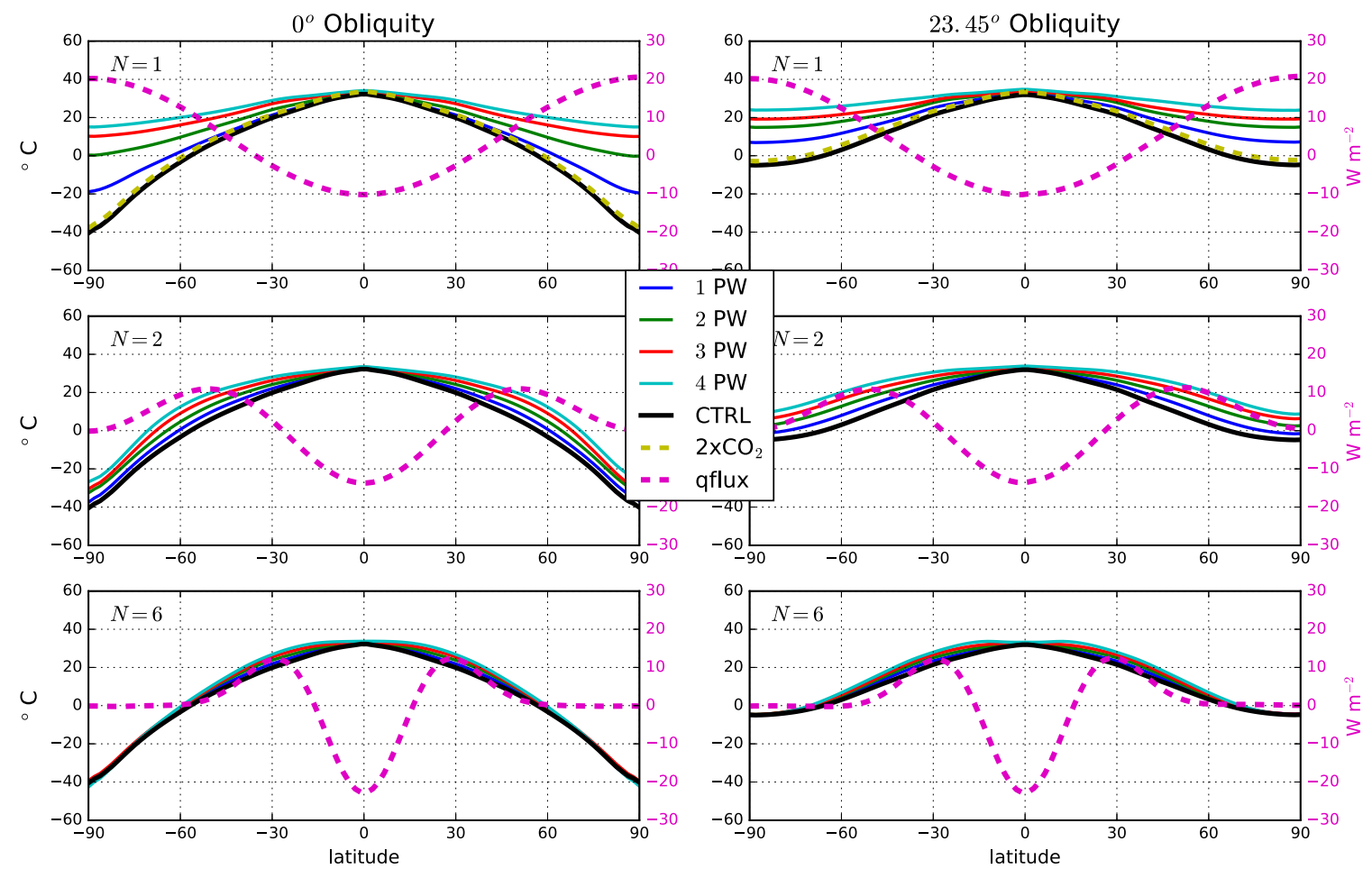

FIG. 2. Zonal, annual mean SST vs latitude as a function of amplitude for (left) $0^{\circ}$ and (right) $23.45^{\circ}$ obliquity. Each panel has a fixed meridional scale parameter $N$ as indicated. The dashed magenta lines show the spatial pattern of the $q$ flux (plotted in W m ${ }^{-2}$ for a $1 \mathrm{PW}$ peak transport). Dashed yellow lines (plotted in the $N=1$ panels only) show the effects of doubling $\mathrm{CO}_{2}$ from the zero-OHT control states.

from Rose and Ferreira (2013), who found polaramplified SST responses for all $N$ in their intermediatecomplexity model.

We can collapse our two-dimensional array of simulations onto a single axis by plotting global mean SST anomalies versus OHT across $30^{\circ}$ latitude, as shown in the upper panels of Fig. 3. With the exception of $N=1$, the results fall onto a single line with a slope of roughly $2 \mathrm{~K} \mathrm{PW}^{-1}$. That is, a $1 \mathrm{PW}$ increase of the OHT out of the tropics results in a global mean warming of $2 \mathrm{~K}$, regardless of the detailed spatial pattern of the OHT convergence (slightly more at $23.45^{\circ}$ obliquity than at $0^{\circ}$ ). The sensitivity is substantially higher for $N=1$, in which the polar sea surface is directly heated. As mentioned previously, the sensitivity to doubled $\mathrm{CO}_{2}$ is 1.71 and $1.97 \mathrm{~K}$ for $0^{\circ}$ and $23.45^{\circ}$ obliquity, respectively. Globally, a $1 \mathrm{PW}$ increase in OHT is roughly equivalent to doubling $\mathrm{CO}_{2}$. However, the warming mechanisms are completely different, as we will show.

The lower panels of Fig. 3 illustrate the dependence of warming patterns on spatial structure of OHT. The contoured quantity is the SST anomaly for a $1 \mathrm{PW}$ increase in OHT from 1 to $2 \mathrm{PW}$, with latitude on the $x$ axis and the meridional scale parameter $N$ on the $y$ axis (and linear interpolation between simulations). The solid black lines show the location of the peak heating, which lies at lower latitudes for larger $N$. The dashed line at the equator represents the peak energy sink. We refer to this as a "spatial anomaly plot" and use this graphical convention repeatedly hereafter.

Figure 3 emphasizes that the warming maximum is collocated with the latitude of OHT convergence, and that this warming increases monotonically as the convergence (i.e., the heating) shifts poleward. In the deep tropics, changes are minimal regardless of the spatial pattern of the forcing. This implies a universal response to increased OHT in the deep tropics and a spatially dependent response in the midlatitudes. We will look into possible mechanisms in the next section.

We found that the changes in SST, as well as the mechanisms driving them, are largely insensitive to the magnitude of the prescribed OHT. For brevity, we will only analyze the anomalies for the most realistic case, an increase in OHT magnitude from 1 to $2 \mathrm{PW}$. Our conclusions will hold for the other prescribed transport patterns.

\section{b. Effects of obliquity}

As a quick aside, it is worth mentioning the similarities in the warming patterns associated with obliquity 

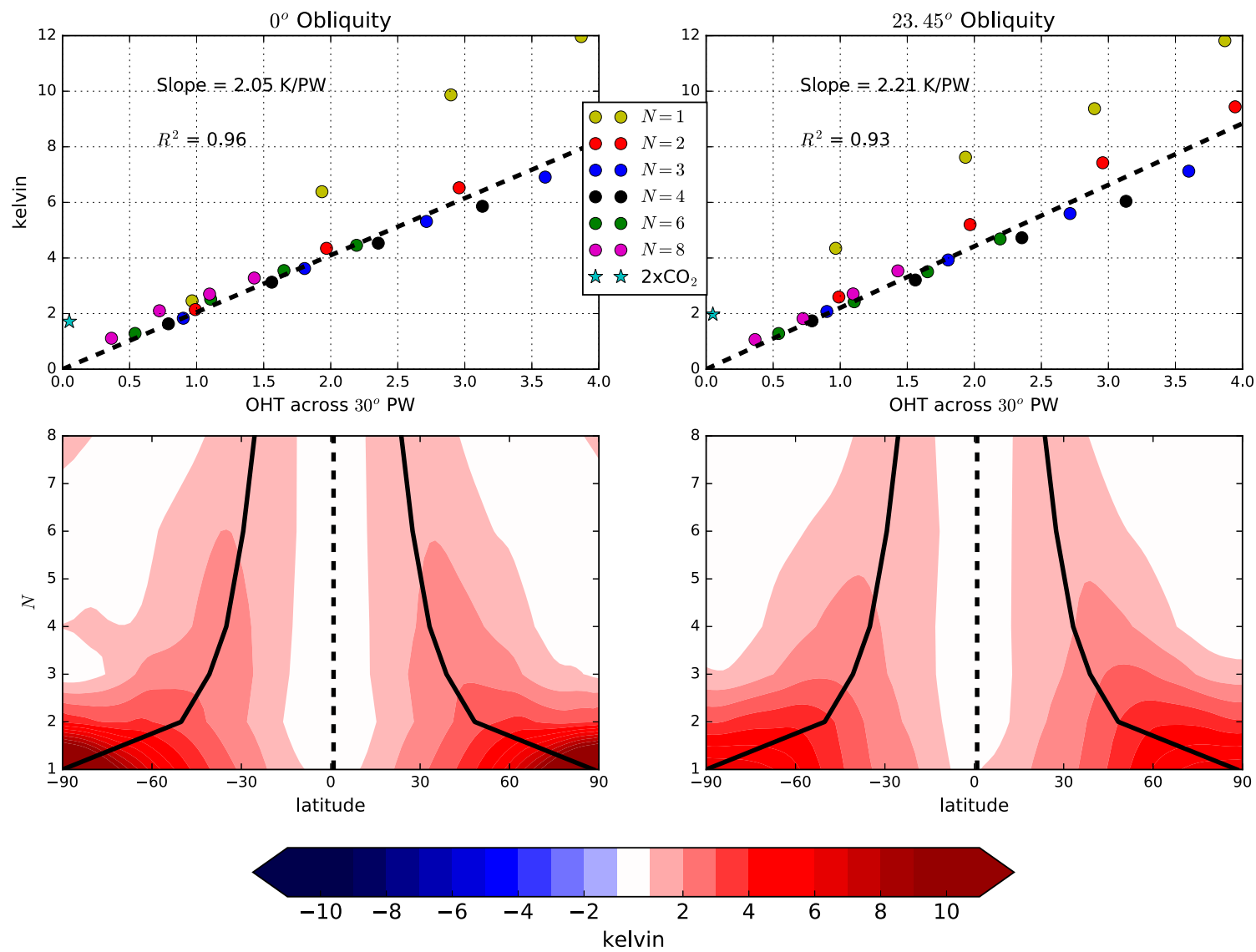

FIG. 3. (top) Global mean SST anomalies with dashed linear best-fit lines and (bottom) "spatial anomaly plots" of SST (lower) for the (left) $0^{\circ}$ and (right) $23.45^{\circ}$ obliquity cases. The contoured quantity is the SST anomaly for a $1 \mathrm{PW}$ increase in OHT (from 1 to $2 \mathrm{PW}$ peak transport). Solid and dashed lines show latitudes of maximum OHT convergence and divergence, respectively.

and OHT. Increased obliquity from $0^{\circ}$ to $23.45^{\circ}$ also elicits a global mean warming and a decrease in the equator-to-pole temperature gradient, shown in Fig. 4. This figure also shows the inferred energy transport associated with both the change in annual-mean insolation and surface shortwave flux.

Increased obliquity shifts energy from the tropics to the high latitudes, qualitatively similar to an increase in OHT. Looking first at the prescribed TOA forcing, the inferred insolation transport in Fig. 4 is similar to our $2 \mathrm{PW}, N=1$ OHT pattern, although the obliquity-induced warming $(3.13 \mathrm{~K})$ is only about half as large. This muted response can be attributed to the reflective nature of optically thick clouds. Taking into account the local albedo, we find that the inferred energy transport associated with the shortwave (SW) flux reaching the surface is about half of that of the insolation, better matching the global mean warming.

With that said, and excluding the outlying $N=1$ case, we have found very little difference in climatic response to $\mathrm{OHT}$ variations at $0^{\circ}$ and $23.45^{\circ}$ obliquity. Just as with the variations in the magnitude of OHT, we will only present results from the $0^{\circ}$ simulations hereafter. Our conclusions will hold for $23.45^{\circ}$ obliquity.

\section{c. Evaporation and hydrological cycle}

Figure 5 shows hydrological cycle changes associated with increased OHT. The drying out of the equator and the moistening of the subtropics are opposite to the "wet

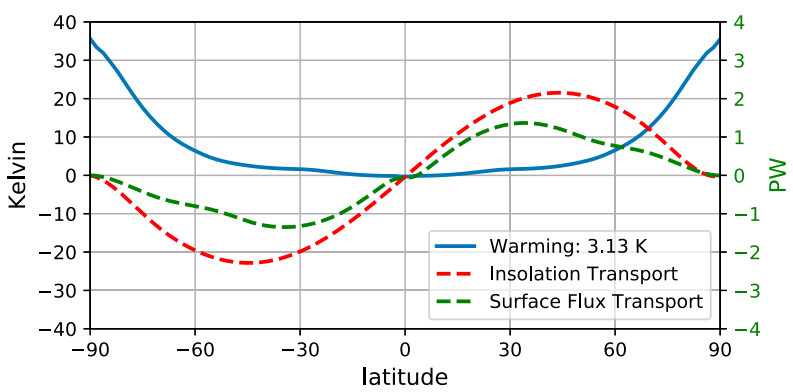

FIG. 4. Anomalies in SST, insolation (expressed as a meridional transport), and annual-mean SW flux reaching the surface (expressed as a meridional transport) for an obliquity increase from $0^{\circ}$ to $23.45^{\circ}$. Global mean warming is $3.13 \mathrm{~K}$. 

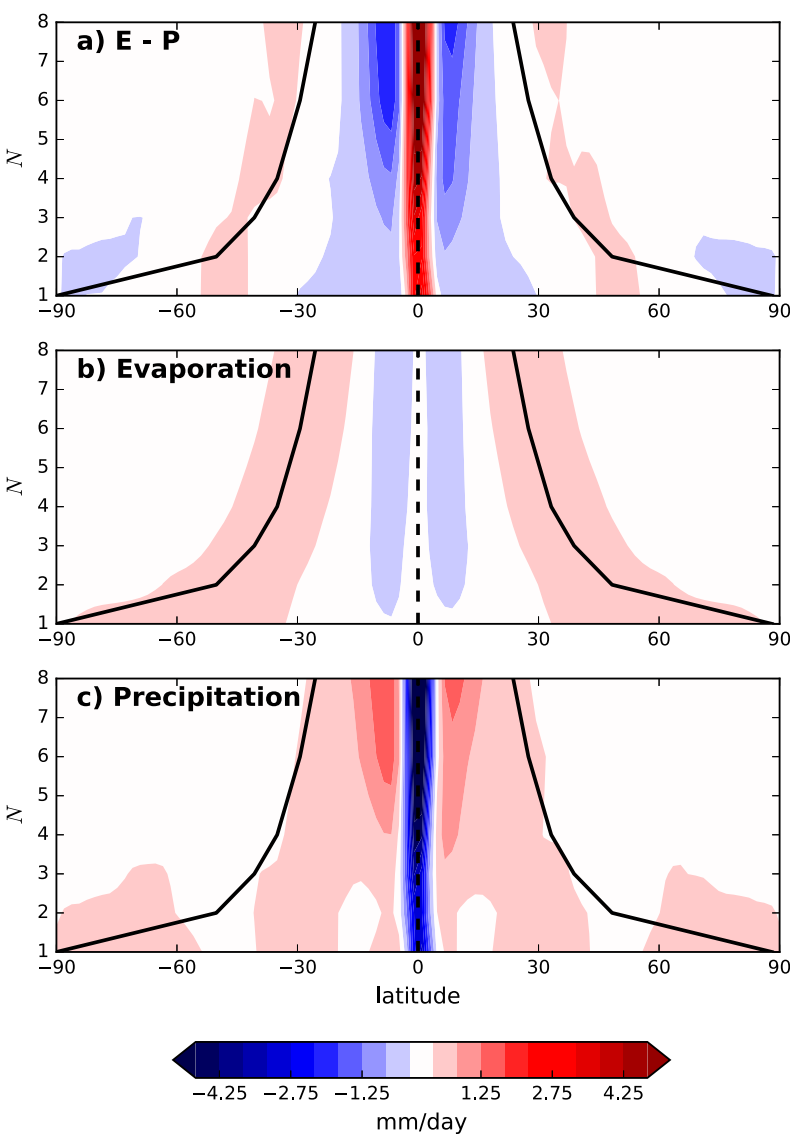

FIG. 5. Spatial anomaly plots (for a $1 \mathrm{PW}$ increase in OHT from 1 to $2 \mathrm{PW}$, as in Fig. 3, plotted for $0^{\circ}$ obliquity only) of (a) $E-P$, (b) evaporation, and (c) precipitation in $\mathrm{mm} \mathrm{day}^{-1}$.

gets wetter, dry gets drier" paradigm for a doubling of $\mathrm{CO}_{2}$ (Held and Soden 2006), emphasizing the difference between the two warming mechanisms. Net subtropical evaporation $(E-P)$ decreases with OHT-driven global warming on the order of $-7 \mathrm{~cm} \mathrm{yr}^{-1} \mathrm{~K}^{-1}\left(-0.2 \mathrm{~mm} \mathrm{day}^{-1} \mathrm{~K}^{-1}\right)$. This is qualitatively similar to the findings of Rose and Ferreira (2013), but about half the rate.
In the absence of other factors such as changes in nearsurface relative humidity and the surface-atmosphere temperature difference, evaporation should increase with SST at the Clausius-Clapeyron (CC) rate of roughly $7 \% \mathrm{~K}^{-1}$. This helps explain the band of increased evaporation collocated with the maximum warming and OHT convergence. However, Fig. 5 also shows robust bands of decreased evaporation straddling the equator for all $N$, which must deviate from the $\mathrm{CC}$ rate given the lack of cooling at these locations. Understanding what is driving these evaporative changes may provide insight into the tropical thermostat mechanism.

Following the method of Lorenz et al. (2010), we decompose the fractional rates of change of evaporation into additive contributions from various physical processes represented in the bulk formula for surface evaporation (details in appendix B). Figure 6 shows this breakdown for both a doubled $\mathrm{CO}_{2}$ scenario and an increase in OHT from 1 to $2 \mathrm{PW}$ with $N=4$. Solid black lines show total deviations from the $\mathrm{CC}$ rate (sum of the colored solid lines). Red lines show contributions from air-sea temperature difference (evaporation will increase if SST warms faster than the near-surface air temperature). Blue lines show the relative humidity (RH) contribution (evaporation rates decrease in response to increased boundary layer RH). Purple lines show contributions from changes in the turbulent exchange coefficients, with the component directly attributable to surface wind speed changes shown in dashed purple.

The doubled $\mathrm{CO}_{2}$ scenario shows a fairly uniform $-5 \% \mathrm{~K}^{-1}$ deviation from the $\mathrm{CC}$ rate, with all three terms contributing to this reduction. This is consistent with the expectation of about $2 \% \mathrm{~K}^{-1}$ increase in evaporation under $\mathrm{CO}_{2}$-driven warming (Held and Soden 2006). Results for increased OHT are completely different. Across the deep tropics the change is near $-25 \% \mathrm{~K}^{-1}$ below the $\mathrm{CC}$ rate (yielding an absolute
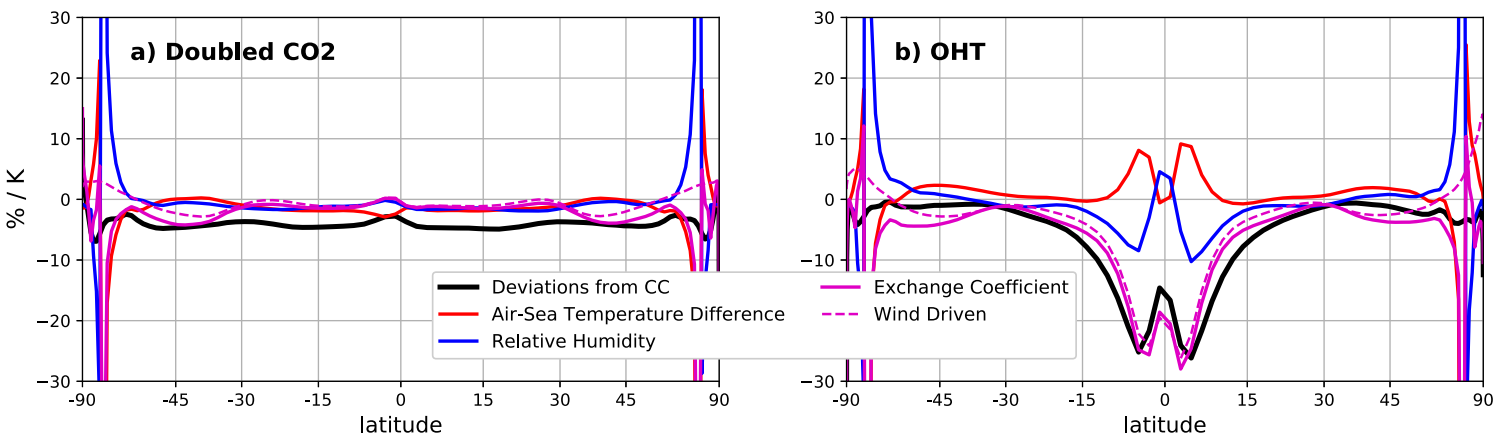

FIG. 6. Breakdown of the contributions to surface evaporation anomalies, expressed as deviations of the fractional evaporation changes away from the $\mathrm{CC}$ rate (roughly $7 \% \mathrm{~K}^{-1}$ ) following the method of Lorenz et al. (2010) (left) for doubled $\mathrm{CO}_{2}$ and (right) a $1 \mathrm{PW}$ increase in OHT for $N=4$ (plotted for $0^{\circ}$ obliquity). 
rate of change of about $-10 \%$ to $-20 \% \mathrm{~K}^{-1}$ ). Our decomposition identifies reduced near-surface wind speed as the key factor in this large reduction in evaporation, with second-order and partially canceling contributions from increased air-sea temperature difference and increased RH. As we will show below, the slower wind speeds are a manifestation of a slowdown in the overturning rate of the Hadley cells (HC). In the midlatitudes, deviations from the $\mathrm{CC}$ rate are much smaller and of similar order to the changes under doubled $\mathrm{CO}_{2}$.

Though we have only plotted a single OHT pattern in Fig. $6(N=4)$, the results are relatively robust across all our simulations. Increased OHT causes a substantial reduction in the surface evaporation [and thus of the surface cooling through the latent heat flux (LHF)] across the deep tropics, associated with a slowdown of the surface winds. This is a possible mechanism for the tropical thermostat, in agreement with Koll and Abbot (2013).

Returning to Fig. 5, the largest hydrological changes are for precipitation: strong reduction at the equator and increases off the equator. These are also associated with a slowdown of the HC, with reduced equatorial convection and subtropical subsidence. Increased convection on either side of the equator is magnified by surface warming associated with OHT convergence for high $N$. In agreement with Rose and Ferreira (2013), there is also a shift from large-scale stratiform to convective precipitation in the storm track regions for low $N$ (not shown).

\section{d. Atmospheric compensation}

The hydrological cycle changes shown in Figs. 5 and 6 indicate that large-scale tropical circulation changes play a role in setting the SST response to increased OHT. These same circulation changes may also impact poleward heat transport. Figure 7 shows the total THT, AHT, and OHT transport for all simulations. While increasing OHT out of the tropics does result in a compensating decrease in AHT, this compensation is incomplete as THT does increase slightly (up to $1 \mathrm{PW}$ ). Despite this, latitudinal shifts in the peak of the THT are nonexistent despite variations in the spatial pattern of the forcing. This is in line with Stone (1978), which predicted the presence of a transport peak near $35.3^{\circ}$ that is also insensitive to partitioning changes. It is also worth noting that in some of the more extreme cases, AHT reverses and moves energy equatorward. We find that these states are characterized by a prominent double ITCZ, resulting in secondary circulation features in the upper troposphere transporting dry static energy equatorward.

To better understand how this partial compensation is accomplished, we decompose AHT into changes associated with the MMC and the transient eddy component
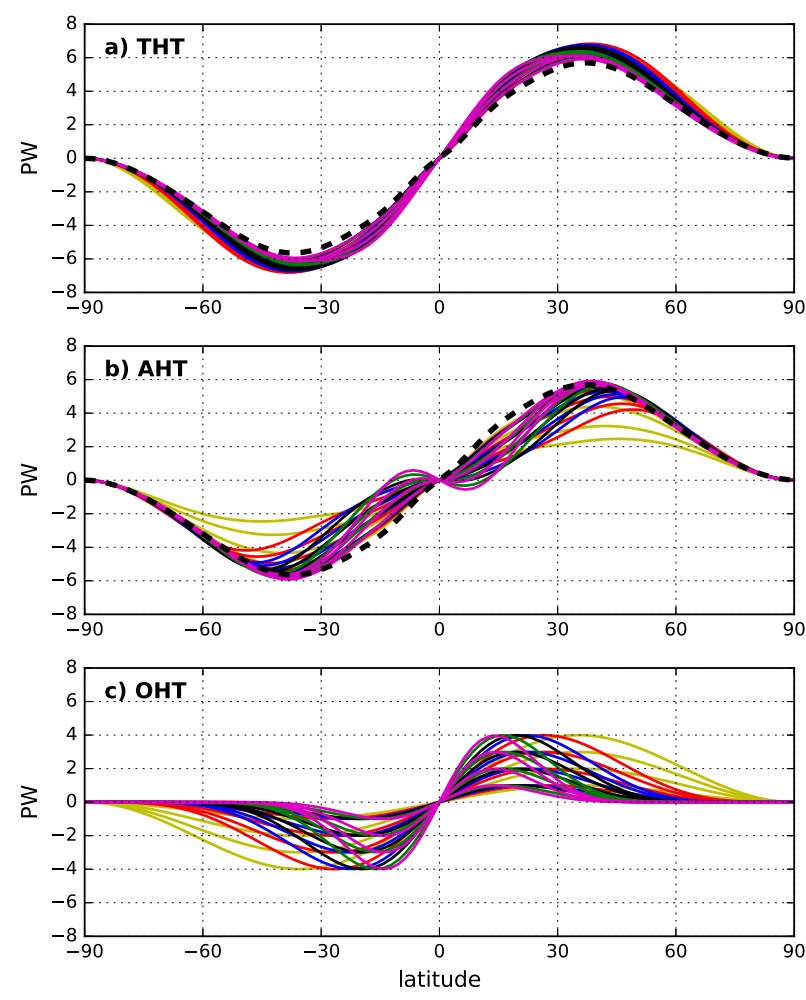

FIG. 7. Zonally averaged (a) THT, (b) AHT, and (c) OHT for all runs with line colors grouped by $N$. The dashed black line is the control THT and AHT (plotted for $0^{\circ}$ obliquity).

(Trenberth and Stepaniak 2003; Hill et al. 2015). AHT is calculated as

$$
F_{\mathrm{tot}}(\phi)=2 \pi a^{2} \int_{-\frac{\pi}{2}}^{\phi}\left(\left[\bar{Q}_{\mathrm{TOA}}\right]-\left[\bar{Q}_{\mathrm{sfc}}\right]\right) \cos \phi d \phi
$$

with $Q_{\mathrm{TOA}}$ and $Q_{\mathrm{sfc}}$ the downward energy flux at the TOA and surface, $a$ the planetary radius, and the square brackets and overbars representing zonal and time mean, respectively. The MMC component of this flux is calculated as

$$
F_{\mathrm{HC}}(\phi)=2 \pi a \cos \phi \int_{P_{\mathrm{top}}}^{P_{\mathrm{scc}}}[\bar{m}][\bar{v}]_{\mathrm{adj}} \frac{d p}{g},
$$

with $m$ the MSE, $v_{\text {adj }}$ the meridional wind adjusted for mass imbalance over the entire column following Hill et al. (2015), and $P_{\text {top }}$ and $P_{\text {sfc }}$ as the pressure at the tropopause and surface, respectively. Because stationary eddies are negligible on a zonally symmetric aquaplanet, the transient eddy component is simply taken to be the residual between (3) and (4).

Results are plotted in Fig. 8. The AHT adjustment is largely a local response to OHT variations, in the sense that changes in AHT tend to be equal and opposite to 

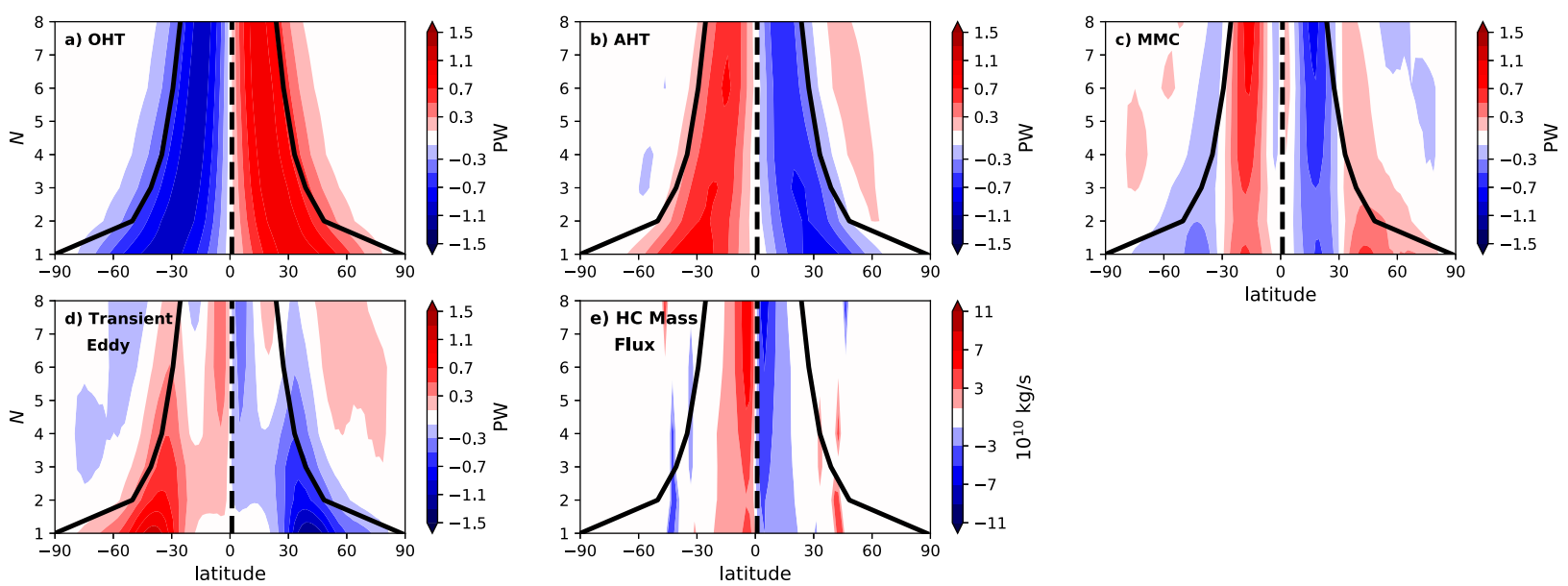

FIG. 8. Spatial anomaly plots (as in Fig. 3, for $0^{\circ}$ obliquity only) for (a) total OHT, (b) total AHT, (c) the transient eddy component of AHT, (d) the MMC component of AHT, and (e) the HC mass flux.

changes in OHT and thus occur farther poleward for small $N$. The primary mechanism for this compensation shifts from MMC for large $N$ to transient eddies for small $N$. This is perhaps not surprising, as the $\mathrm{HC}$ and transient eddies are largely tropical and midlatitude phenomena, respectively. However, the tropical decrease in the MMC component is robust for all $N$.

In the tropics, then, the majority of the AHT decrease can be attributed to changes in the HC. This is partially accomplished through the physical slowdown of the cell, which can be measured through its mass flux. We compute $\psi_{\max }$, the signed maximum of the Eulerian meridional mass streamfunction $(\psi)$, from

$$
\psi(\phi, p)=2 \pi a \cos \phi \int_{0}^{p}[\bar{v}]_{\mathrm{adj}} \frac{d p}{g} .
$$

Figure 8 shows a decrease in the mass flux for all $N$. This implies that an increase in OHT, regardless of its detailed spatial structure, results in a compensating decrease in the AHT partially achieved by a slowdown in the HC. It is important to note that this slowdown is robust across all $N$, implying that many of the conclusions regarding the climatic impacts of the HC slowdown in past works (Koll and Abbot 2013) as well as below are also robust to the spatial structure of OHT. We will analyze the dynamical and thermodynamical mechanisms driving this robust $\mathrm{HC}$ slowdown in future work.

\section{e. TOA energy budget and clouds}

Increasing OHT out of the tropics results in a compensating decrease in AHT, partially accomplished through the slowdown of the HC. But how do these circulation changes contribute to weaker equator-topole temperature gradients, and what, if anything, is amplifying the warming at mid- to high latitudes? Here we examine aspects of the energy budgets at TOA and the surface to gain some insight into these questions.

Figure 9 shows changes in TOA radiative fluxes along with the surface LHF. There is a net TOA energy flux into the tropics and out of the midlatitudes for increased OHT, amplified in the polar regions. This increased meridional gradient is associated with the slightly increased THT found in all runs. There are large, partially compensating changes in TOA longwave (LW) and SW radiation budgets at the equator across all $N$, resulting in the net energy flux into the climate system. On either side of the equator there is a slight increase in the LW flux. These compensating changes are also present in the midlatitudes, amplified in the storm track regions for low $N$. The polar regions are characterized by a decrease in both the LW and SW energy fluxes, resulting in the net deficit. The LW anomalies result from a combination of cloud and clear-sky greenhouse trapping changes. The SW anomalies, on the other hand, are almost entirely dictated by cloud changes since the surface albedo is fixed in our ice-free aquaplanet simulations. These SW changes are nearly universally positive across our ensemble of simulations, indicating a robust loss of clouds in response to increased OHT. Changes in the surface LHF mirror the evaporation anomalies from Fig. 6.

Figure 10 shows cloud fraction changes, decomposed into high, mid, and low cloud. Equatorial cloud cover decreases at all levels in all cases, a consequence of the weakening $\mathrm{HC}$ and reduced equatorial convection. Away from the equator, the largest changes are found in the low clouds. In the subtropics, reduced subsidence associated with HC slowdown results in near-uniform 

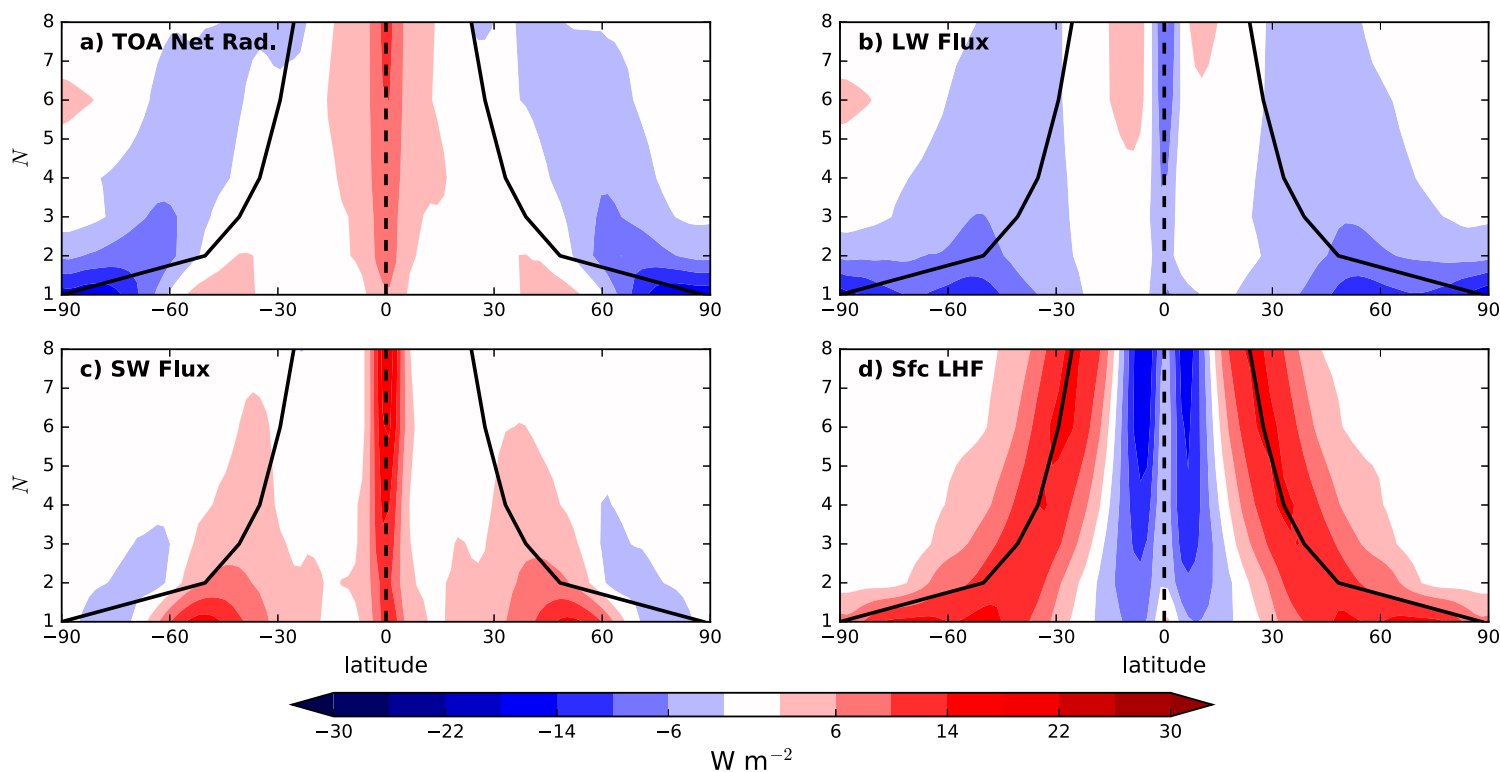

FIG. 9. Spatial anomaly plots (as in Fig. 3, for $0^{\circ}$ obliquity only) of (a) TOA net radiative flux, (b) TOA LW radiative flux, (c) TOA SW radiative flux, and (d) SFC LHF. The sign convention is positive down at TOA for (a)-(c) and positive up at the surface for (d).

reduction of low clouds for all $N$. In the midlatitudes, the dominant effect appears to be decreased lowertropospheric stability associated with the sea surface heating for small $N$, which tends to eliminate the stable inversions that sustain low cloud cover (Wood and Bretherton 2006). For high $N$, the destabilizing effect of subtropical surface warming invigorates local convection, driving the increase in mid and high clouds. Results for the polar region are mixed, with decreased low clouds for all but the $N=1$ case. Overall, Fig. 10 shows that global cloudiness decreases with increased OHT, and the effects are largest for small $N$ (large-scale OHT). The climatic impacts of these cloud changes will be quantified in the next section.
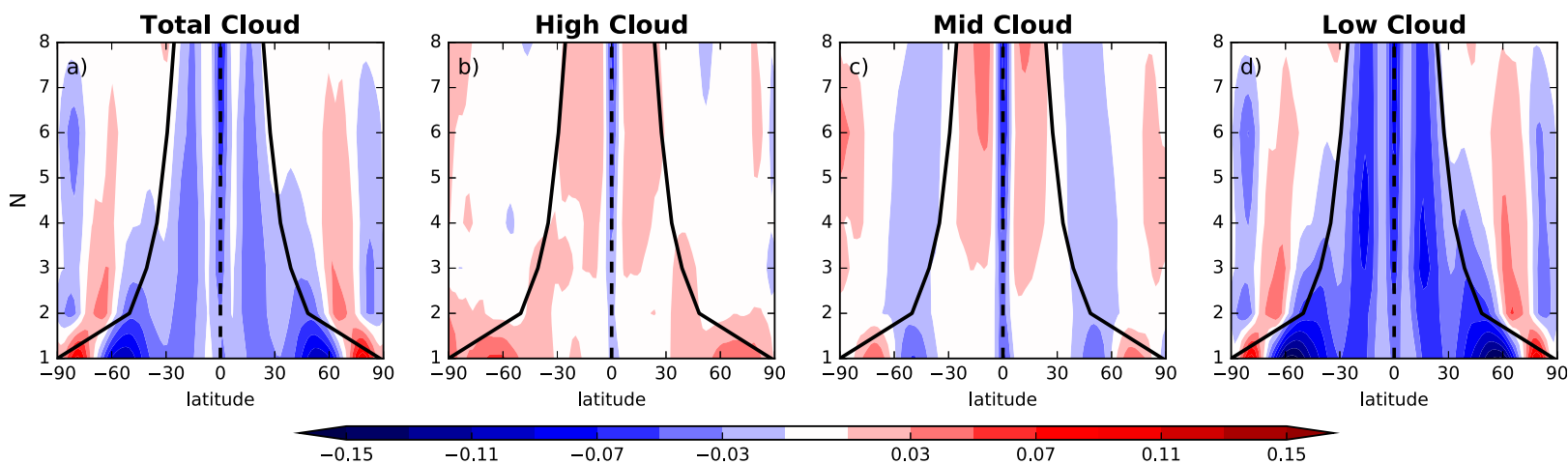

The increased LW flux to space shown in Fig. 9 is consistent with the warming climate but also includes effects of any changes in greenhouse trapping (e.g., by water vapor or clouds) that may be causing the warming. To help disentangle these, in Fig. 11 we plot changes in greenhouse trapping, defined as the difference between surface emissions and emissions to space:

$$
G_{t}=\varepsilon_{s} \sigma T_{s}^{4}-\mathrm{OLR}
$$

where $\sigma$ is the Stefan-Boltzmann constant and $\varepsilon_{s}=0.98$ is the surface emissivity prescribed in the GCM. Positive $\Delta G_{t}$ indicates an enhanced greenhouse effect that contributes to the surface warming. We decompose $\Delta G_{t}$ into additive

FIG. 10. Cloud fraction anomalies (as in Fig. 3, for $0^{\circ}$ obliquity only), vertically integrated for the (a) total atmosphere, (b) high cloud fraction anomalies vertically integrated for cloud top pressures (CTP) less than $440 \mathrm{hPa}$, (c) mid cloud anomalies vertically integrated for CTP between 680 and $440 \mathrm{hPa}$, and (d) low cloud anomalies vertically integrated for CTP greater than $680 \mathrm{hPa}$. 

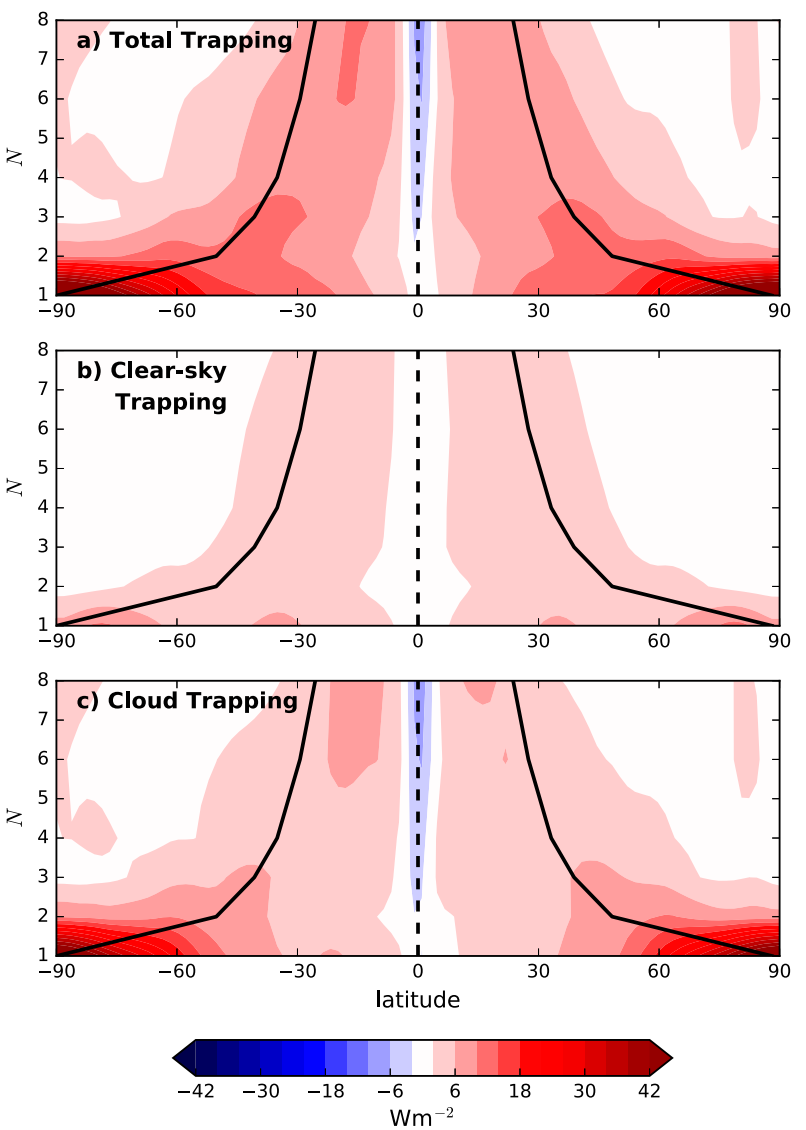

FIG. 11. Anomalies of greenhouse trapping (as in Fig. 3, for $0^{\circ}$ obliquity only), decomposed into (a) total, (b) clear-sky, and (c) cloud components.

clear-sky and cloudy-sky changes $\Delta G_{t}^{\text {clear }}, \Delta G_{t}^{\text {cloudy }}$ as detailed in appendix C. Importantly, our clear-sky trapping diagnostic $\Delta G_{t}^{\text {clear }}$ is not simply (6) computed with the clearsky LW flux; rather, it is reduced by the climatological clear-sky fraction at each location, so that it represents actual contributions of clear-sky processes to changes in trapping (see appendix C).

From Fig. 11, $\Delta G_{t}$ decreases slightly at the equator and an increase pretty much everywhere else, with the maximum collocated with the latitude of OHT convergence, and amplified in the polar regions for low $N$. Parameter $\Delta G_{t}^{\text {clear }}$ is small but positive across broad swaths of the subtropics and midlatitudes, indicating a moistening of the atmosphere with warming. Parameter $\Delta G_{t}^{\text {cloudy }}$ is negative (decreased trapping) at the equator but positive everywhere else, and typically larger than $\Delta G_{t}^{\text {clear }}$. This is especially true for small $N$, for which the changes at mid- to high latitudes are very large. Thus, changes in the greenhouse effect of high-latitude clouds are playing a strong role in the polar-amplified warming for small $N$, while clear-sky greenhouse processes are secondary and not as dependent on the spatial structure of OHT.

\section{Diffusive model interpretation}

Results in the previous section strongly suggest that cloud changes play a dominant role in driving the global warming in response to increased OHT. In this section we return to the simple EBM introduced in section 1 with the specific goal of understanding the relative importance of clear-sky feedbacks and cloud changes on the warming patterns.

We extend (2) with an additional radiative forcing term $R(\phi)$ on the rhs representing cloud forcing, and we also allow the clear-sky feedback $\lambda$ to vary with latitude:

$$
C \frac{\partial T}{\partial t}=\lambda(\phi) T(\phi)+H(\phi)+R(\phi)-\nabla \cdot F(\phi) .
$$

The feedback $\lambda(\phi)$ is the sum of the Planck, lapse rate, and water vapor feedbacks, which we calculate by the radiative kernel method (Soden et al. 2008). We use radiative kernels for a zonally symmetric aquaplanet from Feldl and Roe (2013).

We treat the GCM-derived cloud response as an external forcing $R(\phi)$ in the EBM. Specifically, we set $R(\phi)$ equal to the anomalous cloud radiative effect (CRE) associated with a given increase in OHT, where CRE is the difference between all-sky and clear-sky TOA radiative flux. This allows us to isolate the effects of cloud changes on SST (both local and nonlocal) and estimate the hypothetical climatic response to OHT in the absence of cloud feedbacks. Because we are treating the clouds as a forcing rather than a feedback in these calculations, $\lambda$ in (7) is strictly a clear-sky feedback. It is important to note that with this setup, we cannot analyze the influences that variations in the cloud forcing and the clear-sky feedbacks have on each other.

Figure 12 shows the forcing terms $H(\phi)$ and $R(\phi)$ (OHT convergence and associated CRE) for a $1 \mathrm{PW}$ increase in OHT, along with the kernel-derived $\lambda(\phi)$. Parameter $H(\phi)$ is the same forcing that was applied to our baseline EBM and GCM calculations. Consistent with our discussion in section 3e, the CRE is positive almost everywhere, meaning that cloud changes are working to warm the climate. The equatorial signal is associated with the weakening $\mathrm{HC}$ and is relatively insensitive to $N$. The CRE is largest globally for small $N$ for which the loss of midlatitude low cloud and the increased greenhouse trapping of polar clouds both play a role.

The clear-sky feedback (Fig. 12c) is negative (i.e., stabilizing) in most locations. We are especially interested in the dependence of $\lambda(\phi)$ on $N$. If the spatial structure of 

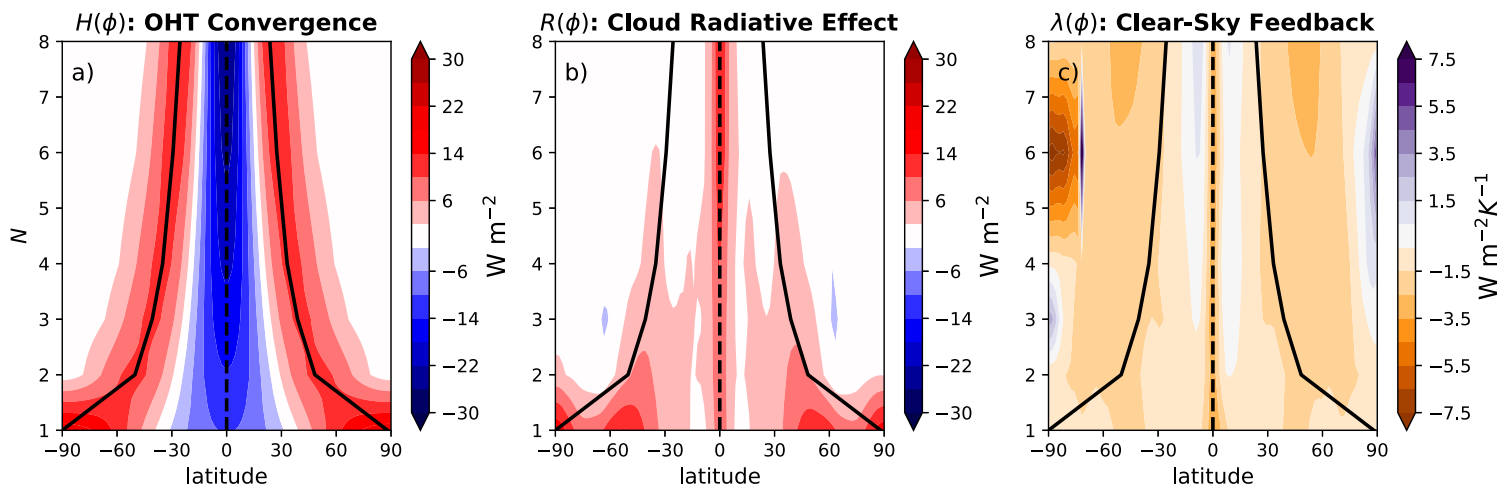

FIG. 12. Spatial anomaly plots (as in Fig. 3, for $0^{\circ}$ obliquity only) of (a) OHT convergence, (b) CRE, and (c) clear-sky feedbacks.

radiatively important water vapor change (for example) were highly dependent on the spatial structure of the imposed OHT, this would presumably show up here as variations in $\lambda(\phi)$. Figure $12 \mathrm{c}$ suggests that variations with $N$ as well as with latitude are modest and probably not of leading-order importance in determining the SST response. The large asymmetry between the North and South Poles for $N=5,6$ can be attributed to noise in the kernel calculation as the change in SST becomes very slightly negative. We will test this assertion in the EBM by comparing two variants of the model: one with the detailed feedback $\lambda(\phi)$, and another with a simple homogeneous feedback $\lambda=-1.45 \mathrm{~W} \mathrm{~m}^{-2} \mathrm{~K}^{-1}$, based on the global average clear-sky feedback diagnosed from the GCM. This is the same constant value used in our baseline EBM.

We also compare two different diffusive parameterizations for the heat transport $F(\phi)$. The classical drydiffusive model (e.g., North 1975a) sets

$$
F_{\mathrm{dry}}(\phi)=-K_{\mathrm{dry}} \frac{d T}{d \phi},
$$

and we use $K=0.55 \mathrm{~W} \mathrm{~m}^{-2} \mathrm{~K}^{-1}$ (our baseline model).

An alternative parameterization crudely incorporates the effects of moisture on the efficiency of atmospheric heat transport:

$$
F_{\text {moist }}(\phi)=-K_{\text {moist }} \frac{d}{d \phi}[T\{1+f(\phi)\}],
$$

where $f(\phi)$ is a moisture amplification factor

$$
f(\phi)=\left.\frac{L r}{c_{p}} \frac{d q *}{d T}\right|_{T_{\mathrm{ret}}(\phi)},
$$

with $r$ the near-surface relative humidity and $d q^{*} / d T$ the rate of change of saturation specific humidity at reference temperature $T_{\text {ref }}$. The parameterization (9) expresses the diffusion of near-surface moist static energy, linearized about the control climate and assuming fixed $r$ (Rose et al. 2014). This parameterization increases the efficiency of diffusion for warm tropical temperatures relative to extratropical temperatures. We set $K_{\text {moist }}=0.24 \mathrm{~W} \mathrm{~m}^{-2} \mathrm{~K}^{-1}$ for the moist case with 0.8 relative humidity, following Hwang and Frierson (2010) and Rose et al. (2014). Parameter $K_{\text {dry }}$ is tuned so that the dry model gives the same peak heat transport for a $1 \mathrm{PW}$ increase in OHT with $N=1$ as the moist model. We will test whether the dry model (8) or the moist model (9) gives a better quantitative fit to the GCM results. Both models are strictly linear, so that responses to $H(\phi)$ and $R(\phi)$ are additive.

The EBM was solved numerically out to equilibrium using the open-source Python package climlab (Rose 2018). Results are plotted in Fig. 13, with the four different model variants in rows and the responses to the two different types of forcing in columns. We also plot the misfit of the EBM solutions to the GCM temperatures. The baseline EBM from section 1 is shown here as the simplest case of no cloud forcing, dry diffusivity, and constant feedback, and is used to validate our numerical EBM solutions against the analytical solution presented in appendix A.

All EBM variants exhibit tropical cooling and extratropical warming in response to increased OHT. The cooling is most pronounced for the most detailed version of the EBM with moist diffusion and local feedbacks. However, the tropical cooling is strongly mitigated by warming from clouds in all cases. This warming effect is tropically amplified for larger $N$ (the diffusive response to the positive equatorial CRE associated with loss of convective cloud), but polar amplified for small $N$ (the response to widespread loss of low clouds in the vicinity of the heated region). The combination of the direct effects of OHT and the warming effect from cloud changes results in reduced equator-to-pole SST gradients and tropical thermostat effects, much like in 

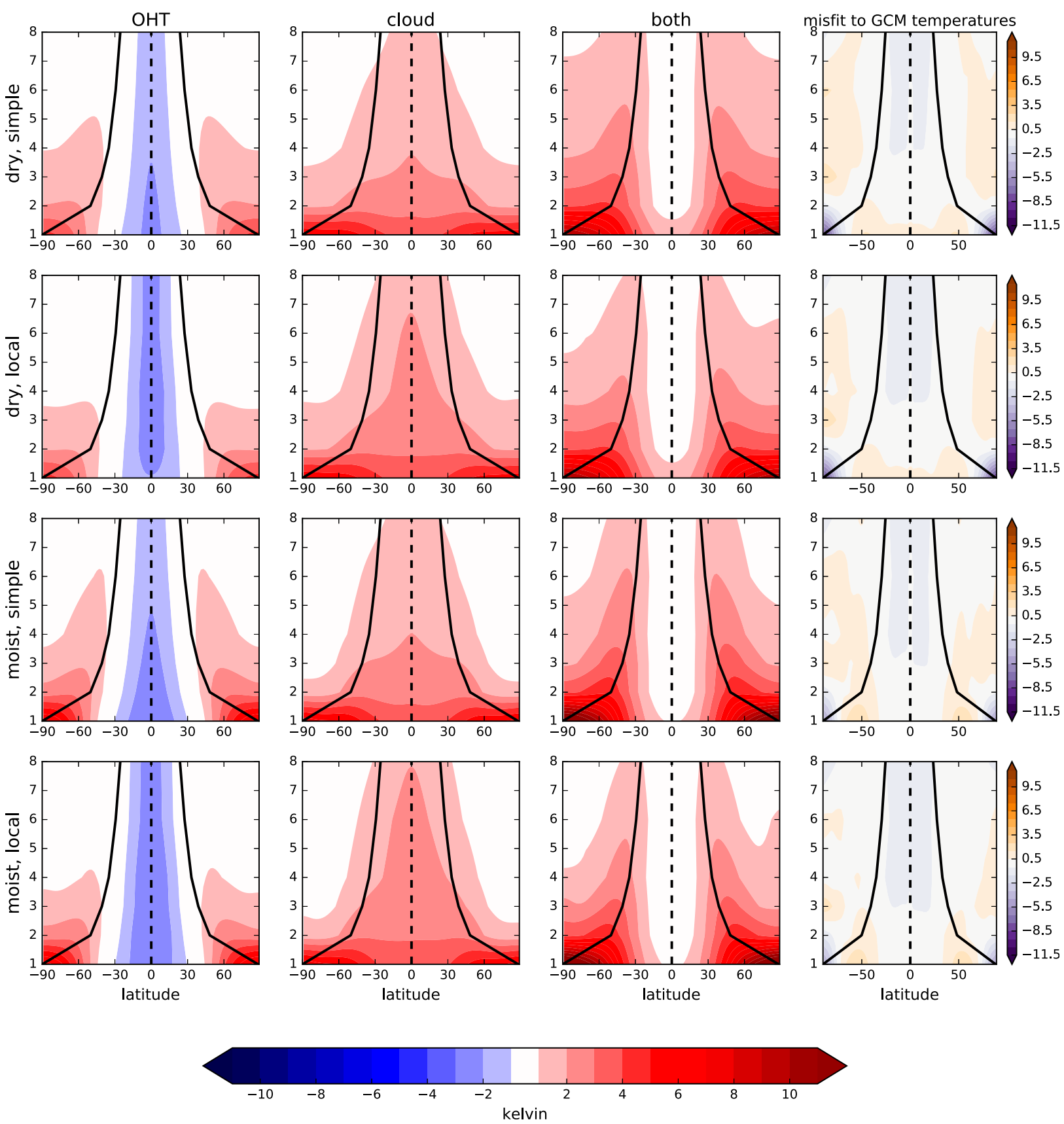

FIG. 13. SST anomalies predicted by the diffusive EBM and comparison to the GCM (at $0^{\circ}$ obliquity). First column shows the cloud-free EBM response to a $1 \mathrm{PW}$ increase in OHT (forcing shown in Fig. 12a). Second column shows the EBM response to prescribed cloudiness changes as diagnosed from the GCM (Fig. 12b). Third column is the response to the combined forcing. Fourth column is the misfit of the EBM solutions to the GCM temperatures. Each row shows solutions for a different set of EBM assumptions-dry or moist diffusion and constant or local clear-sky feedback, as detailed in the text.

Fig. 3. A version of the EBM in which we impose a global-mean cloud response for each $N$ fails to capture this warming pattern (not shown).

Figure 13 also shows that meridional structure in the clear-sky feedbacks and the diffusion approximation have surprisingly little effect on the warming pattern. In the tropics, both variables enhance the cooling associated with OHT divergence and the warming associated with the cloud response, resulting in the negligible net change in SST. The polar amplification for small $N$ is present in all model variants, but is slightly stronger with both moist diffusion and local feedback. 
The good quantitative agreement between the EBM and the GCM confirms that cloud changes play the key role in determining the SST response to a change in OHT. Because the cloud forcing varies strongly with $N$, the mechanisms ultimately driving the warming in the GCM are not universal but depend on the spatial pattern of the OHT.

It is somewhat surprising that the EBM provides such a good fit given that it does not account for changes in wind-driven evaporation. We showed in Fig. 6 that the slowdown of the $\mathrm{HC}$ is accompanied by a dramatic decrease in tropical evaporation, and that the resulting large changes in LHF (Fig. 9d) might be critical to the tropical thermostat mechanism. However, convection in the deep tropics strongly couples the tropospheric temperature to the boundary layer and surface below, meaning that the direct SST response to variations in the latent heat flux (i.e., evaporation) are somewhat muted in the climate system (Pierrehumbert 1995).

However, the absence of this effect might still be responsible for the very small negative misfit in EBM temperatures across the tropics for high values of $N$. Koll and Abbot (2013) shows that the changes in evaporation associated with an increase in OHT result in a slight tropical warming comparable to the misfit in Fig. 13. Also, we have treated cloud changes as an external forcing in the EBM calculation and made no attempt to construct a fully closed model for the effects of increased OHT. It is possible that the climatic impact of reduced evaporation is actually felt most through cloud changes - particularly the sharp reduction in convective clouds at the equator.

\section{Discussion and conclusions}

We have shown that increased OHT in an aquaplanet climate model is associated with warmer temperatures and weaker equator-to-pole temperature gradients. The warming pattern depends on the meridional structure of the OHT, with global mean surface temperatures increasing slightly more than $2 \mathrm{~K} \mathrm{PW}^{-1}$ transport across $30^{\circ}$ latitude. The warmer climate is associated with reduced Hadley circulation, reduced equatorial convection, moister subtropics, reduced cloudiness, and partial but incomplete compensation by AHT. Rather than cooling the tropics, as might be expected from elementary principles (such as our baseline EBM), increased OHT actually warms the tropics slightly. Both the warming pattern and the hydrological cycle changes differ from those driven by $\mathrm{CO}_{2}$, suggesting that drivers of past global change might be detectable from combinations of hydroclimate and temperature proxies.

A comprehensive illustration of these various mechanisms is presented in Fig. 14. Increasing OHT out of the tropics results in a partially compensating decrease in
AHT. This decrease is accomplished in part via the physical slowdown of the $\mathrm{HC}$, resulting in a decrease in equatorial convection. Decreased convection results in an increase in SW radiation at the surface, offsetting the OHT-driven cooling. In the midlatitudes, surface warming via OHT convergence works to destabilize the lower troposphere and reduce low cloud cover. This in turn increases the SW flux into the surface, further amplifying the midlatitude warming.

Herweijer et al. (2005) and Rose and Ferreira (2013) both attributed the warming from increased OHT primarily to midlatitude moistening. However, we have found that clouds, rather than clear-sky greenhouse processes, play the greatest role in the atmospheric adjustment to increased OHT. We attribute these differences to the relatively simple nature of the GCMs used in the previous studies, particularly with regard to their vertical resolutions. The MITgcm used by Rose and Ferreira (2013) and the GISS model primarily used by Herweijer et al. (2005) had 5 and 8 vertical layers respectively compared to the 26 levels in CAM4. The limited resolution meant fewer degrees of freedom for the models to adjust to the surface forcing, resulting in an atmospheric response dominated by deep convection and the moistening of the upper troposphere. The higher vertical resolution of the CESM allows for much greater complexity in the low cloud response as well as better coupling of the moist boundary layer to the troposphere.

The meridional SST response to increased OHT simply cannot be captured without knowing the meridional structure of the cloud response. This cloud response in our GCM involves both a reduction in deep tropical clouds (closely tied to circulation changes) and a widespread loss in boundary layer clouds. The low cloud response in particular is sensitive to the meridional scale of the OHT. Cases for which the convergence is large in the mid- to high latitudes experience the largest loss of low cloud and consequently experience the largest warming. This accounts for the approximate scaling of global mean temperature with OHT across $30^{\circ}$ latitude. Unlike Rose and Ferreira (2013), the warming is not polar amplified except in cases where OHT reaches into the high latitudes. There is no single universal temperature response pattern governing the climatic response to increased OHT.

The tropical thermostat mechanism can be described as a competition between cooling by OHT divergence and warming by clouds, in agreement with Koll and Abbot (2013). The robustness of this thermostat across all spatial patterns of OHT presents some interesting implications with regard to the warm, equable climate problem presented in the introduction. 


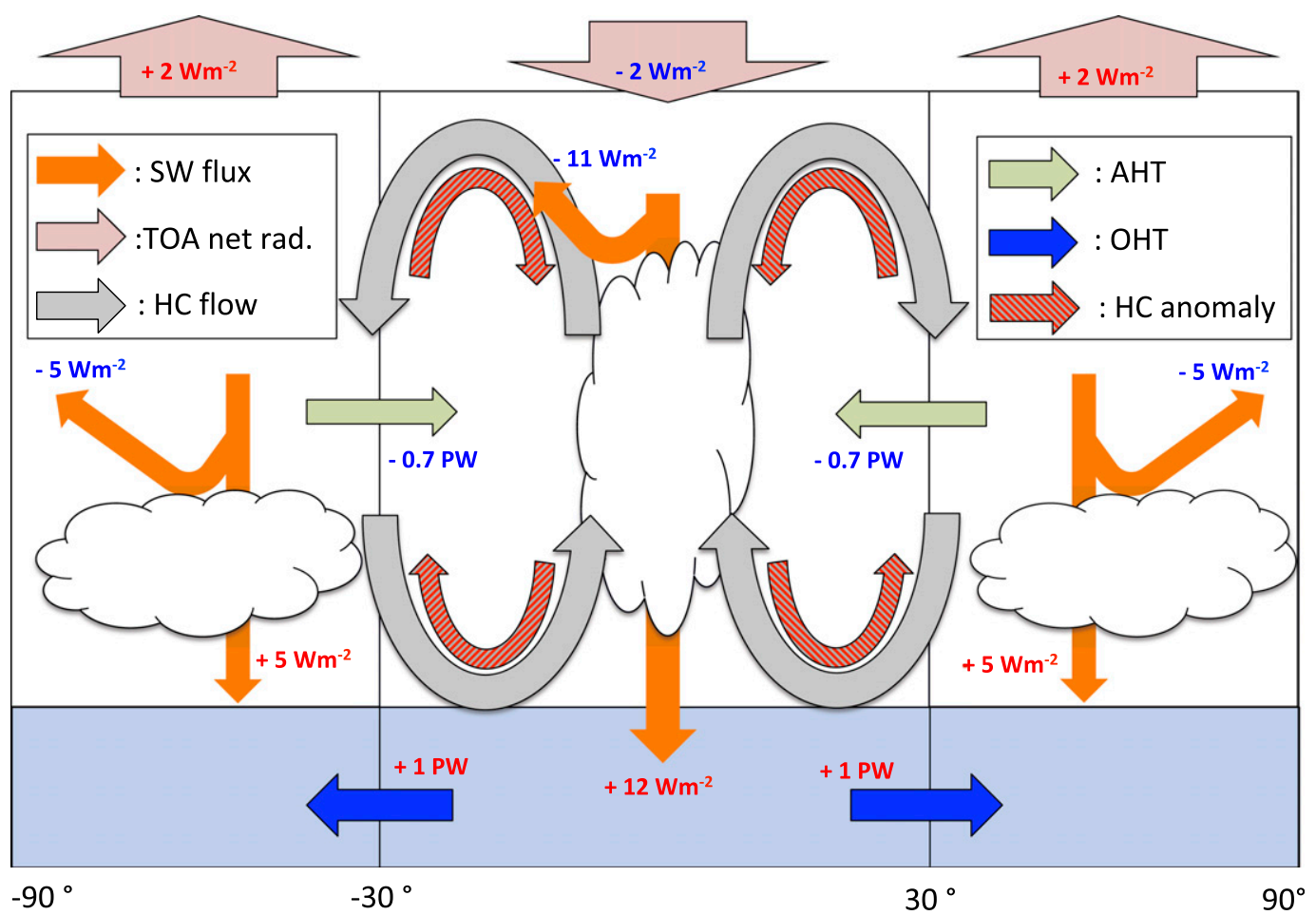

FIG. 14. Schematic representation of the various transport and warming mechanisms associated with a $1 \mathrm{PW}$ increase in OHT for $N=2$. The blue and green arrows represent the change in OHT and AHT, respectively, across $30^{\circ}$. The gray and red shaded lines represent the control and anomalous HC flow, respectively. The light red arrows represent area-average changes in net TOA radiation for the tropical and extratropical boxes. Oranges arrows are not box-averaged values, but instead represent more localized changes in SW flux associated with key cloud changes at the equator and in the midlatitudes.

Even though past OHT patterns are largely unconstrained by proxy data (Bice et al. 2000), this warming mechanism is still viable as the presence of the tropical thermostat does not depend on the forcing pattern. It is a fundamental aspect of the climate response to increased OHT.

The robustness of the Hadley cell slowdown raises interesting questions about the driving dynamical and thermodynamical mechanisms. Are these mechanisms themselves robust across all $N$, and how does the $\mathrm{HC}$ slowdown relate to compensation in heat transport? These questions and others surrounding the $\mathrm{HC}$ will be addressed in a follow-up study.

These results are robust for both $0^{\circ}$ and $23.45^{\circ}$ obliquity patterns. The introduction of seasonality into the model has little influence on the annual-mean response of the HC. It should be noted, however, that the model has weak seasonal temperature cycles due to the $60-\mathrm{m}$ mixed layer depth. There are some differences in the SST response with regard to the amount of polar amplification in the most extreme cases, but they can be attributed to weakened cloud responses at the poles.
It is important to note that these results may be model dependent. While we have shown that there is robustness in the results between the two different obliquity cases within the CAM4 atmospheric GCM, we have yet to test these results using other advanced climate models. Herweijer et al. (2005), Koll and Abbot (2013), and Rose and Ferreira (2013) all look at the effects of increasing the OHT out of the tropics with different AGCMs and produce different results. This can be attributed to the importance of the model water vapor parameterizations and convective schemes to the mechanisms driving the warming. While the warming effects of OHT have been well established, more work is needed to test the robustness of the mechanisms presented in this paper.

Last, we acknowledge that these results are limited by the simplified nature of model setup. Global climatic response to OHT variations is mediated in part by sea ice feedbacks and land-sea interactions, yet both are ignored in these simulations. It is therefore useful to think of the warming as a minimum response for an increase in OHT as surface albedo changes will further amplify midlatitude and polar responses. However, 
there is no evidence that the radiative mechanisms driving the warming patterns will differ greatly in more realistic simulations. The results presented are a crucial step in the hierarchical approach to studying OHT effects on the climate system.

Acknowledgments. We thank Nicole Feldl for providing the aquaplanet radiative kernels used in the EBM analysis. We also thank Chris Colose for fruitful discussions on this topic over the years. We also thank two anonymous reviewers for their thoughtful comments. This work was supported by NSF Award AGS-1455071.

\section{APPENDIX A}

\section{Analytical Solution of the Diffusive EBM}

The diffusive EBM (2) is solvable analytically in its simplest configuration with zero CRE, dry diffusivity, and uniform feedback. The steady-state form of the simple model can be written

$$
K_{\mathrm{dry}} \frac{d}{d x}\left[\left(1-x^{2}\right) \frac{d T}{d x}\right]-|\lambda| T(x)=-H(x),
$$

with $x=\sin \phi$, and where we take the absolute value because of our sign convention $\lambda<0$. As discussed by North (1975b), (A1) is a form of Legendre's equation and is solvable so long as the forcing term $-H(x)$ can be expressed as a series of Legendre polynomials $P_{n}(x)$.

For any forcing term $A_{n} P_{n}(x)$ on the rhs of (A1), there is a solution that satisfies no-flux boundary conditions at the poles:

$$
T_{n}(x)=-\frac{A_{n}}{|\lambda|} \frac{P_{n}(x)}{1+n(n+1) \delta},
$$

with the nondimensional transport efficiency parameter (e.g., Rose et al. 2017)

$$
\delta=\frac{K_{\mathrm{dry}}}{|\lambda|}
$$

from which we can build a full solution for (A1) by expanding $H(x)$ in a Legendre series.

Here $H(x)$ is the OHT convergence in $\mathrm{W} \mathrm{m}^{-2}$. Taking the convergence of (1) and accounting for the spherical geometry gives

$$
H(x)=-\frac{1}{2 \pi a^{2}} \frac{d}{d x}\left[\Psi x\left(1-x^{2}\right)^{N}\right],
$$

$$
=-\frac{\Psi}{2 \pi a^{2}}\left(1-x^{2}\right)^{N-1}\left\{1-x^{2}(1+2 N)\right\},
$$

which is polynomial in $x^{2}$ and can thus be expanded in even-order $P_{n}(x)$ :

$$
H(x)=\frac{\Psi}{2 \pi a^{2}} \sum_{n=2,4, \ldots, 2 N} b_{n} P_{n}(x),
$$

where

$$
\sum_{n=2,4, \ldots, 2 N} b_{n} P_{n}(x)=-\left(1-x^{2}\right)^{N-1}\left\{1-x^{2}(1+2 N)\right\} .
$$

The coefficients $b_{n}$ are computed from the expansion formula for even-order Legendre polynomials $P_{n}(x)$ :

$$
b_{n}=(2 n+1) \int_{0}^{1} F(x) P_{n}(x) d x
$$

where

$$
F(x)=-\left(1-x^{2}\right)^{N-1}\left\{1-x^{2}(1+2 N)\right\} .
$$

Expansion coefficients $b_{n}$ are listed in Table A1 for $N$ from 1 to 8 .

The full solution to (A1) is thus

$$
T(x)=\frac{\Psi}{2 \pi a^{2}|\lambda|} \sum_{n=2,4, \ldots, 2 N} \frac{b_{n}}{1+n(n+1) \delta} P_{n}(x) .
$$

To use this formula to calculate SST anomalies associated with a given change in OHT, we must recognize that the relevant amplitude $\Psi$ depends on $N$. The peak OHT occurs at the latitude of zero convergence:

$$
x_{\max }=\frac{1}{\sqrt{1+2 N}}
$$

which we can plug into (1) to get

$$
\Psi=\mathrm{OHT}_{\max } \frac{(1+2 N)^{N+1 / 2}}{(2 N)^{N}} .
$$

Setting $\mathrm{OHT}_{\max }=1 \mathrm{PW}$ in (A12), substituting in (A10) and using the expansion coefficients in Table A1 then gives a formula for the SST anomaly associated with a $1 \mathrm{PW}$ increase in OHT for a given $N$ in the simple EBM with constant feedbacks and dry diffusion. These solutions are plotted in the top-left panel of Fig. 13 and validate the numerical solver used for the rest of Fig. 13. 
TABLE A1. Expansion of OHT convergence $-d / d x\left\{x\left(1-x^{2}\right)^{N}\right\}=\sum_{n=2,4},{ }_{2 N} b_{n} P_{n}(x)$ in even-order Legendre polynomials for various values of $N$.

\begin{tabular}{|c|c|c|c|c|c|c|c|c|}
\hline$N$ & $b_{2}$ & $b_{4}$ & $b_{6}$ & $b_{8}$ & $b_{10}$ & $b_{12}$ & $b_{14}$ & $b_{16}$ \\
\hline 1 & 2 & & & & & & & \\
\hline \multirow[t]{2}{*}{2} & $\frac{8}{7}$ & $-\frac{8}{7}$ & & & & & & \\
\hline & & $\overline{7}$ & & & & & & \\
\hline \multirow{2}{*}{3} & 176 & 288 & 16 & & & & & \\
\hline & $\overline{231}$ & $\overline{231}$ & $\overline{33}$ & & & & & \\
\hline \multirow{2}{*}{4} & 128 & 1152 & 128 & 128 & & & & \\
\hline & $\overline{231}$ & $\overline{1001}$ & $\overline{165}$ & $\overline{715}$ & & & & \\
\hline \multirow{2}{*}{5} & 1280 & 1024 & 512 & 1024 & 256 & & & \\
\hline & $\overline{3003}$ & $\overline{1001}$ & $\overline{561}$ & $\overline{2717}$ & $\overline{4199}$ & & & \\
\hline \multirow{2}{*}{6} & 1024 & 15360 & 10240 & 10240 & 15360 & 1024 & & \\
\hline & $\overline{3003}$ & $\overline{17017}$ & $\overline{10659}$ & $\overline{19019}$ & $\overline{96577}$ & $\overline{52003}$ & & \\
\hline \multirow{2}{*}{7} & 2048 & 36864 & 10240 & 40960 & 129024 & 4096 & 2048 & \\
\hline & $\overline{7293}$ & $\overline{46189}$ & $\overline{10659}$ & $\overline{62491}$ & $\overline{482885}$ & $\overline{66861}$ & $\overline{334305}$ & \\
\hline \multirow{2}{*}{8} & 32768 & -32768 & 229376 & -229376 & 1605632 & -229376 & 229376 & -32768 \\
\hline & $\overline{138567}$ & $\overline{46189}$ & $\overline{245157}$ & $\overline{312455}$ & $\overline{4345965}$ & $\overline{1938969}$ & $\overline{10363455}$ & $\overline{17678835}$ \\
\hline
\end{tabular}

\section{APPENDIX B}

\section{Evaporation Analysis}

Following Lorenz et al. (2010), we analyze changes in evaporation through a simple bulk formula:

$$
E=k\left\{q^{*}\left(T_{s}\right)-r q^{*}\left(T_{a}\right)\right\},
$$

where $E$ is evaporation; $q^{*}(T)$ is the saturation specific humidity; $r$ is the near-surface relative humidity; $k=\rho C|\mathbf{U}|$ is the turbulent exchange coefficient that depends on wind speed and static stability; and $T_{s}, T_{a}$ represent SST and near-surface air temperatures, respectively. Taking the fractional derivative with respect to $T_{a}$ gives [Eq. (9) from Lorenz et al. (2010)]

$$
\begin{aligned}
\frac{d \ln E}{d T_{a}}=\alpha-\frac{(1-\gamma) \alpha q^{*}\left(T_{s}\right)}{q^{*}\left(T_{s}\right)-r q^{*}\left(T_{a}\right)} & \frac{d r}{d T_{a} q^{*}\left(T_{a}\right)} \\
& -\frac{d \ln k}{q^{*}\left(T_{s}\right)-r q^{*}\left(T_{a}\right)}+\frac{d T_{a}}{d T_{a}}, \\
\frac{d \ln k}{d T_{a}}= & \frac{d \ln (\rho C)}{d T_{a}}+\frac{d \ln |\mathbf{U}|}{d T_{a}},
\end{aligned}
$$

where $\alpha=1 / q^{*} d q^{*} / d T$ is the CC rate (approximately $7 \% \mathrm{~K}^{-1}$ ) and $\gamma=d T_{s} / d T_{a}$ is the ratio of changes in SST and near-surface air temperature.

Figure 6 shows additive contributions to deviations from the CC rate $d \ln E / d T_{a}-\alpha$ from the second, third, and fourth terms on the lhs of (B2). The second term measures the effects of changes in air-sea temperature difference and is positive if SST warms at a faster rate than $T_{a}$ (i.e., $\gamma>1$ ). The third term measures the effects of near-surface relative humidity changes and is positive if warming is accompanied by a decrease in RH. The fourth term measures the effect of the changes in the turbulent exchange coefficient and can be further decomposed into changes in near-surface wind speed and static stability through (B3). A decrease in wind speed with warming results tends to suppress evaporation below the $\mathrm{CC}$ rate.

\section{APPENDIX C}

\section{Clear-Sky and Cloudy-Sky Contributions to Greenhouse Trapping}

Greenhouse trapping $G_{t}$ as defined in (6) is a number in $\mathrm{W} \mathrm{m}^{-2}$ that measures the amount by which surface emissions are reduced due to the presence of a longwave-absorbing atmosphere. We can readily calculate changes in $G_{t}$ after adjustment to some climate forcing in a climate model:

$$
\Delta G_{t}=\Delta F_{s}^{\uparrow}-\Delta \mathrm{OLR},
$$

and this diagnostic has been used in a number of papers (e.g., Bony et al. 1997; Herweijer et al. 2005; Barreiro et al. 2011; Rose and Ferreira 2013).

Here we seek the most meaningful way to decompose $\Delta G_{t}$ into cloud and noncloud effects, a point that has not been addressed clearly in the literature. For any given region with cloud fraction $c$, the total flux to space is an average of the flux from clear and cloudy sky:

$$
\mathrm{OLR}=(1-c) \mathrm{OLR}^{\text {clear }}+c \mathrm{OLR}^{\text {cloudy }},
$$

so that small changes from a control climate can be conceptually decomposed as 


$$
\begin{aligned}
\Delta \mathrm{OLR} \approx & (1-c) \Delta \mathrm{OLR}^{\text {clear }}+c \Delta \mathrm{OLR}^{\text {cloudy }} \\
& +\Delta c\left(\mathrm{OLR}^{\text {cloudy }}-\mathrm{OLR}^{\text {clear }}\right)
\end{aligned}
$$

and $\Delta F_{s}^{\uparrow}$ can also be conceptually decomposed into clear-sky and cloudy components:

$$
\Delta F_{s}^{\uparrow}=(1-c) \Delta F_{s}^{\uparrow}+c \Delta F_{s}^{\uparrow} .
$$

This is useful because $(1-c) \Delta F_{s}^{\uparrow}$ actually represents the contribution of clear-sky regions to the total change in upwelling flux.

The total trapping change can thus be written

$$
\begin{aligned}
\Delta G_{t}= & (1-c) \Delta G_{t}^{*}+c\left[\Delta F_{s}^{\uparrow}-\Delta \operatorname{OLR}^{\text {cloudy }}\right. \\
& \left.-\frac{\Delta c}{c}\left(\mathrm{OLR}^{\text {cloudy }}-\mathrm{OLR}^{\text {clear }}\right)\right]
\end{aligned}
$$

where we have defined

$$
\Delta G_{t}^{*}=\Delta F_{s}^{\uparrow}-\Delta \mathrm{OLR}^{\text {clear }},
$$

which is the change in trapping that would have occurred under completely clear skies, all else being equal.

The first term in $(\mathrm{C} 5)$, that is, $(1-c) \Delta G_{t}^{*}$, is the actual contribution of clear-sky regions to $\Delta G_{t}$. The second term is related purely to cloud changes (both absorption changes in cloudy regions and changes in cloud fraction). Thus, (C5) gives a natural additive decomposition into clear-sky and cloud processes:

$\Delta G_{t}^{\text {clear }}=(1-c) \Delta G_{t}^{*}$,

$\Delta G_{t}^{\text {cloud }}=c\left[\Delta F_{s}^{\uparrow}-\Delta \mathrm{OLR}^{\text {cloudy }}-\frac{\Delta c}{c}\left(\mathrm{OLR}^{\text {cloudy }}-\mathrm{OLR}^{\text {clear }}\right)\right]$.

However, OLR ${ }^{\text {cloudy }}$ is not a quantity that is directly modeled, so (C8) is not useful in this form. On the other hand, GCMs do routinely calculate OLR ${ }^{\text {clear }}$ (by making a second pass through the radiation code with the clouds zeroed out), so we can compute $\Delta G_{t}^{*}$ from (C6). Parameter $\Delta G_{t}^{\text {cloud }}$ can thus be calculated as a residual using the control climate cloud fraction $c$. Specifically,

$$
\Delta G_{t}^{\text {cloud }}=c \Delta G_{t}^{*}+\Delta \mathrm{OLR}^{\text {clear }}-\Delta \mathrm{OLR},
$$

where the sum of the last two terms is also the LW contribution to the change in CRE. We use (C7) and (C94) for our additive decomposition in Fig. 11.

It is important to make the distinction between $\Delta G_{t}^{*}$, which measures the trapping the would occur if the same climate change occurred with no clouds present, from the quantity $\Delta G_{t}^{\text {clear }}$ which is scaled by the control clear-sky fraction $(1-c)$, and is thus a more useful measure of the actual contribution of clear-sky greenhouse processes to the climate change that occurred.

\section{REFERENCES}

Barreiro, M., A. Cherchi, and S. Masina, 2011: Climate sensitivity to changes in ocean heat transport. J. Climate, 24, 5015-5030, https://doi.org/10.1175/JCLI-D-10-05029.1.

Barron, E. J., 1987: Eocene equator-to-pole surface ocean temperatures: A significant climate problem? Paleoceanography, 2, 729-739, https://doi.org/10.1029/PA002i006p00729.

Bice, K. L., C. R. Scotese, D. Seidov, and E. J. Barron, 2000: Quantifying the role of geographic change in cenozoic ocean heat transport using uncoupled atmosphere and ocean models. Palaeogeogr. Palaeoclimatol. Palaeoecol., 161, 295-310, https://doi.org/10.1016/S0031-0182(00)00072-9.

Bitz, C., M. Holland, E. Hunke, and R. Moritz, 2005: Maintenance of the sea-ice edge. J. Climate, 18, 2903-2921, https://doi.org/ 10.1175/JCLI3428.1.

Bjerknes, J., 1964: Atlantic air-sea interaction. Advances in Geophysics, Vol. 10, Academic Press, 1-82, https://doi.org/10.1016/ S0065-2687(08)60005-9.

Blackburn, M., and B. J. Hoskins, 2013: Context and aims of the aqua-planet experiment. J. Meteor. Soc. Japan, 91A, 1-15, https://doi.org/10.2151/jmsj.2013-A01.

Bony, S., K.-M. Lau, and Y. C. Sud, 1997: Sea surface temperature and large-scale circulation influences on tropical greenhouse effect and cloud radiative forcing. J. Climate, 10, 2055-2077, https:// doi.org/10.1175/1520-0442(1997)010<2055:SSTALS > 2.0.CO;2.

Caballero, R., and P. Langen, 2005: The dynamic range of poleward energy transport in an atmospheric general circulation model. Geophys. Res. Lett., 32, L02705, https://doi.org/ 10.1029/2004GL021581.

Covey, C., and E. Barron, 1988: The role of ocean heat transport in climatic change. Earth-Sci. Rev., 24, 429-445, https://doi.org/ 10.1016/0012-8252(88)90065-7.

Crowley, T., and J. C. Zachos, 2000: Comparison of zonal temperature profiles for past warm time periods. Warm Climates in Earth History, Cambridge University Press, 50-76, doi:10.1017/CBO9780511564512.004.

Czaja, A., and J. Marshall, 2006: The partitioning of poleward heat transport between the atmosphere and ocean. J. Atmos. Sci., 63, 1498-1511, https://doi.org/10.1175/JAS3695.1.

Eisenman, I., 2010: Geographic muting of changes in the arctic sea ice cover. Geophys. Res. Lett., 37, L16501, https://doi.org/ 10.1029/2010GL043741.

Enderton, D., and J. Marshall, 2009: Explorations of atmosphereocean-ice climates on an aquaplanet and their meridional energy transports. J. Atmos. Sci., 66, 1593-1611, https://doi.org/ 10.1175/2008JAS2680.1.

Feldl, N., and G. H. Roe, 2013: The nonlinear and nonlocal nature of climate feedbacks. J. Climate, 26, 8289-8304, https://doi.org/ 10.1175/JCLI-D-12-00631.1.

Ferrari, R., and D. Ferreira, 2011: What processes drive the ocean heat transport? Ocean Modell., 38, 171-186, https://doi.org/ 10.1016/j.ocemod.2011.02.013.

Ferreira, D., J. Marshall, and J.-M. Campin, 2010: Localization of deep water formation: Role of atmospheric moisture transport and geometrical constraints on ocean circulation. J. Climate, 23, 1456-1476, https://doi.org/10.1175/2009JCLI3197.1. 
$\longrightarrow,-$, and B. E. J. Rose, 2011: Climate determinism revisited: Multiple equilibria in a complex climate model. J. Climate, $\mathbf{2 4}$, 992-1012, https://doi.org/10.1175/2010JCLI3580.1.

Greenwood, D. R., and S. L. Wing, 1995: Eocene continental climates and latitudinal temperature gradients. Geology, 23, 1044-1048, https://doi.org/10.1130/0091-7613(1995)023<1044: ECCALT $>2.3 . \mathrm{CO} ; 2$.

Held, I. M., 2001: The partitioning of the poleward energy transport between the tropical ocean and atmosphere. J. Atmos. Sci., 58, 943-948, https://doi.org/10.1175/1520-0469(2001)058<0943: TPOTPE $>2.0$.CO 2 .

- 2005: The gap between simulation and understanding in climate modeling. Bull. Amer. Meteor. Soc., 86, 1609-1614, https://doi.org/10.1175/BAMS-86-11-1609.

_ , and B. J. Soden, 2006: Robust responses of the hydrological cycle to global warming. J. Climate, 19, 5686-5699, https:// doi.org/10.1175/JCLI3990.1.

Herweijer, C., R. Seager, M. Winton, and A. Clement, 2005: Why ocean heat transport warms the global mean climate. Tellus, 57A, 662-675, https://doi.org/10.3402/tellusa.v57i4.14708.

Hill, S., Y. Ming, and I. M. Held, 2015: Mechanisms of forced tropical meridional energy flux change. J. Climate, 28, 1725 1742, https://doi.org/10.1175/JCLI-D-14-00165.1.

Hotinski, R. M., and J. R. Toggweiler, 2003: Impact of a Tethyan circumglobal passage on ocean heat transport and "equable" climates. Paleoceanography, 18, 1007, https://doi.org/10.1029/ 2001PA000730.

Huber, M., and L. C. Sloan, 2001: Heat transport, deep waters, and thermal gradients: Coupled simulation of an Eocene greenhouse climate. Geophys. Res. Lett., 28, 3481-3484, https:// doi.org/10.1029/2001GL012943.

Hwang, Y.-T., and D. Frierson, 2010: Increasing atmospheric poleward energy transport with global warming. Geophys. Res. Lett., 37, L24807, https://doi.org/10.1029/ 2010GL045440.

Jeevanjee, N., P. Hassanzadeh, S. A. Hill, and A. Sheshadri, 2017: A perspective on climate model hierarchies. J. Adv. Model. Earth Syst., 9, 1760-1771, https://doi.org/10.1002/2017MS001038.

Koll, D. B., and D. S. Abbot, 2013: Why tropical sea surface temperature is insensitive to ocean heat transport changes. J. Climate, 26, 6742-6749, https://doi.org/10.1175/JCLI-D-13-00192.1.

Langen, P. L., and V. A. Alexeev, 2004: Multiple equilibria and asymmetric climates in the CCM3 coupled to an oceanic mixed layer with thermodynamic sea ice. Geophys. Res. Lett., 31, L04201, https://doi.org/10.1029/2003GL019039.

Lee, M.-I., M. J. Suarez, I.-S. Kang, I. M. Held, and D. Kim, 2008: A moist benchmark calculation for atmosphere general circulation models. J. Climate, 21, 4934-4954, https://doi.org/10.1175/2008JCLI1891.1.

Lorenz, D. J., E. T. DeWeaver, and D. J. Vimont, 2010: Evaporation change and global warming: The role of net radiation and relative humidity. J. Geophys. Res., 115, D20118, https:// doi.org/10.1029/2010JD013949.

Manabe, S., 1969: Climate and the ocean circulation: II. The atmospheric circulation and the effects of heat transfer by ocean currents. Mon. Wea. Rev., 97, 775-805, https://doi.org/10.1175/ 1520-0493(1969)097<0775:CATOC $>2.3$.CO;2.

Medeiros, B., and B. Stevens, 2011: Revealing differences in GCM representations of low clouds. Climate Dyn., 36, 385-399, https://doi.org/10.1007/s00382-009-0694-5.

Neale, R. B., J. Richter, S. Park, P. H. Lauritzen, S. J. Vavrus, P. J. Rasch, and M. Zhang, 2013: The mean climate of the Community Atmosphere Model (CAM4) in forced SST and fully coupled experiments. J. Climate, 26, 5150-5168, https:// doi.org/10.1175/JCLI-D-12-00236.1.

North, G. R., 1975a: Analytical solution to a simple climate model with diffusive heat transport. J. Atmos. Sci., 32, 1301-1307, https://doi.org/10.1175/1520-0469(1975)032<1301: ASTASC $>2.0 . \mathrm{CO} ; 2$.

_ 1975b: Theory of energy-balance climate models. J. Atmos. Sci., 32, 2033-2043, https://doi.org/10.1175/1520-0469(1975)032<2033: TOEBCM $>2.0 . \mathrm{CO} ; 2$.

Pearson, P. N., and M. Palmer, 2000: Atmospheric carbon dioxide concentrations over the past 60 million years. Nature, 406, 695-699, https://doi.org/10.1038/35021000.

Pierrehumbert, R., 1995: Thermostats, radiator fins, and the local runaway greenhouse. J. Atmos. Sci., 52, 1784-1806, https://doi.org/ 10.1175/1520-0469(1995)052<1784:TRFATL > 2.0.CO; 2 .

Rose, B. E. J., 2015: Stable "waterbelt" climates controlled by tropical ocean heat transport: A nonlinear coupled climate mechanism of relevance to snowball earth. J. Geophys. Res. Atmos., 120, 1404 1423, https://doi.org/10.1002/2014JD022659.

2018: Climlab: A python toolkit for interactive, processoriented climate modeling. J. Open Source Software, 3 (24), 659, https://doi.org/10.21105/joss.00659.

- , and D. Ferreira, 2013: Ocean heat transport and water vapor greenhouse in a warm equable climate: A new look at the low gradient paradox. J. Climate, 26, 2117-2136, https://doi.org/ 10.1175/JCLI-D-11-00547.1.

— , and M. C. Rencurrel, 2016: The vertical structure of tropospheric water vapor: Comparing radiative and ocean-driven climate changes. J. Climate, 29, 4251-4268, https://doi.org/ 10.1175/JCLI-D-15-0482.1.

_ D. Ferreira, and J. Marshall, 2013: The role of oceans and sea ice in abrupt transitions between multiple climate states. J. Climate, 26, 2862-2879, https://doi.org/10.1175/JCLI-D-12-00175.1.

—, K. Armour, D. Battisti, N. Feldi, and D. Koll, 2014: The dependence of transient climate sensitivity and radiative feedbacks on the spatial pattern of ocean heat uptake. Geophys. Res. Lett., 41, 1071-1078, https://doi.org/10.1002/2013GL058955.

, T. W. Cronin, and C. M. Bitz, 2017: Ice caps and ice belts: The effects of obliquity on ice-albedo feedback. Astrophys. J., 846, 28, https://doi.org/10.3847/1538-4357/aa8306.

Seager, R., D. Battisti, J. Yin, N. Gordon, N. Naik, A. Clement, and M. Cane, 2002: Is the Gulf Stream responsible for Europe's mild winters? Quart. J. Roy. Meteor. Soc., 128, 2563-2586, https://doi.org/10.1256/qj.01.128.

Singh, H. A., P. J. Rasch, and B. E. J. Rose, 2017: Increased ocean heat convergence into the high latitudes with $\mathrm{CO}_{2}$ doubling enhances polar-amplified warming. Geophys. Res. Lett., 44, 10 583-10 591, https://doi.org/10.1002/2017GL074561.

Soden, B. J., I. M. Held, R. Colman, K. M. Shell, J. T. Kiehl, and C. A. Shields, 2008: Quantifying climate feedbacks using radiative kernels. J. Climate, 21, 3504-3520, https://doi.org/ 10.1175/2007JCLI2110.1.

Stone, P. H., 1978: Constraints on dynamical transports of energy on a spherical planet. Dyn. Atmos. Oceans, 2, 123-139, https:// doi.org/10.1016/0377-0265(78)90006-4.

Trenberth, K. E., and J. M. Caron, 2001: Estimates of meridional atmosphere and ocean heat transports. Climate Dyn., 14, 3433-3443, https://doi.org/10.1175/1520-0442(2001)014<3433: EOMAAO $>2.0 . \mathrm{CO} ; 2$.

, and D. P. Stepaniak, 2003: Covariability of components of poleward atmospheric energy transports on seasonal and interannual timescales. J. Climate, 16, 3691-3705, https://doi.org/ 10.1175/1520-0442(2003)016<3691:COCOPA > 2.0.CO;2. 
Voigt, A., and Coauthors, 2016: The tropical rain belts with an annual cycle and continent model intercomparison project: TRACMIP. J. Adv. Model. Earth Syst., 8, 1868-1891, https:// doi.org/10.1002/2016MS000748.

Winton, M., 2003: On the climatic impact of ocean circulation. J. Climate, 16, 2875-2889, https://doi.org/10.1175/ 1520-0442(2003)016<2875:OTCIOO >2.0.CO;2.

Wood, R., and C. S. Bretherton, 2006: On the relationship between stratiform low cloud cover and lower-tropospheric stability. J. Climate, 19, 6425-6432, https://doi.org/10.1175/ JCLI3988.1.
Wunsch, C., 2005: The total meridional heat flux and its oceanic and atmospheric partition. J. Climate, 18, 4374-4380, https:// doi.org/10.1175/JCLI3539.1.

Yang, H., Y. Zhao, Z. Liu, Q. Li, F. He, and Q. Zhang, 2015: Heat transport compensation in atmosphere and ocean over the past 22,000 years. Sci. Rep., 5, 16661, https://doi.org/10.1038/ srep16661.

Zhang, G. J., and N. A. McFarlane, 1995: Sensitivity of climate simulations to the parameterization of cumulus convection in the $\mathrm{Ca}$ nadian Climate Centre general circulation model. Atmos.-Ocean, 33, 407-446, https://doi.org/10.1080/07055900.1995.9649539. 